\title{
Zu jung oder zu alt für eine Lehre? Altersdiskriminierung bei der Ausbildungsplatzvergabe
}

\author{
Christian Imdorf
}

Angenommen: 29. August 2011 / Online publiziert: 20. September 2011

(C) Institut für Arbeitsmarkt- und Berufsforschung 2011

Zusammenfassung Zwei konträre Sachverhalte prägen gegenwärtig die Problematisierung des Alters beim Eintritt in die duale Berufsausbildung: Einerseits stellen Ausbildungsbetriebe zunehmend ältere Auszubildende ein, andererseits erhalten zu alte Bewerber Probleme, überhaupt noch einen Ausbildungsplatz zu finden. Die Studie fragt nach den betrieblichen Beweggründen, bei der Auswahl von Auszubildenden die untere Altersgrenze nicht $\mathrm{zu}$ tief $\mathrm{zu}$ halten und gleichzeitig eine obere Altersgrenze durchzusetzen. Mit Bezugnahme auf die französische Soziologie der Konventionen und einem daran orientierten Modell der Personalselektion wurde diese Frage sekundäranalytisch untersucht. Dazu wurden Experteninterviews mit Ausbildungsverantwortlichen aus 60 Deutschschweizer Kleinbetrieben argumentationsanalytisch ausgewertet. Die Resultate belegen, dass ein verzögerter Lehrbeginn mehrheitlich positiv beurteilt wird, und dass manche Betriebe Bewerber abweisen, die als $\mathrm{zu}$ alt gelten. Die Netzwerkkonvention der betrieblichen Koordination führt zu Vorbehalten gegenüber zu jungen Auszubildenden im Hinblick auf gelingende Kundenbeziehungen. Älteren Bewerbern wird dagegen auf dem Hintergrund der häuslichen Konvention die soziale Passung in die Betriebe abgesprochen. Zudem erwarten Betriebe bei älteren Auszubildenden unter Bezugnahme auf die Marktkonvention ein erhöhtes Lehrabbruchsrisiko mit Kostenfolgen. Die rekonstruierte Altersdiskriminierung bei der betrieblichen Ausbildungsstellenvergabe lässt darauf schließen, dass sowohl der verzögerte Eintritt von Schulabgängern in Ausbildung

Zusätzliche Information ist in der online Version dieses Beitrags (doi:10.1007/s12651-011-0093-3) enthalten.

C. Imdorf $(\bowtie)$

Institut für Soziologie, Universität Basel, Petersgraben 27, 4051 Basel, Schweiz

e-mail: christian.imdorf@unibas.ch als auch die Probleme älterer Bewerber bei der Lehrstellensuche durch multiple betriebliche Koordinationsanforderungen mitbedingt sind.

Schlüsselwörter Ausbildungsbetriebe .

Lehrlingsselektion · Konventionen · Alter · Diskriminierung

JEL Klassifikationen J71 · M51 · M53 · Z13

Too-young or too-old for an apprenticeship? Age discrimination in hiring apprentices

Abstract Two contrary effects determine how age influences access to apprenticeships: on the one hand, training companies increasingly hire older apprentices. On the other hand applicants who are too old, face difficulty in finding an apprenticeship place at all. The paper analysis the selecting companies' motives in setting both the upper and lower age limits for new recruits. Referring to a conceptual framework of personnel selection drawn from the French sociology of conventions, the research question is investigated using secondary data analysis. Expert interviews with personnel managers in 60 small businesses from German-speaking Switzerland were interpreted using argument analysis. The findings show that most of the analysed training companies favour applicants who have not directly left school, but have usually spent one year in some kind of tiding-over arrangement. Despite this preference for 'older' candidates a significant minority of companies turns down applicants who have already reached a certain age. The network convention of workplace coordination gives rise to reservations towards too-young apprentices with regard to customer relations. In contrast, firms turn down older applicants because they believe they do not socially 'fit in', which can be interpreted through the domestic convention of coordination. 
These applicants are associated with a higher dropout risk, which would additionally entail costs to the firm in terms of the market convention. Having revealed the operations of age discrimination in hiring apprentices, the paper concludes that both the school leavers' delayed start of training as well as the problems faced by older job applicants to access apprenticeships are in part caused by training companies and their multiple requirements for social coordination.

Keywords Training company · Apprentice selection . Conventions $\cdot$ Age $\cdot$ Discrimination

\section{Einleitung}

In der Schweiz und in Deutschland ist die duale Ausbildung in Betrieb und Berufsschule die überwiegende Form der Berufsausbildung. Aufgrund ihres direkten Bezugs zur Arbeitswelt gilt diese Form der Ausbildung als ein Erfolgsmodell, das im internationalen Vergleich durch tiefe Jugendarbeitslosigkeitsquoten besticht. Zahlreiche Untersuchungen zeigen jedoch, dass in den letzten Jahrzehnten der Übergang von der Sekundarstufe I in die Berufsausbildung für viele Ausbildungssuchende schwieriger geworden ist (für einen Überblick vgl. Hupka-Brunner et al. 2011). Das Risiko eines verzögerten Übergangs in die betriebliche Berufsausbildung ist für Jugendliche mit unzureichenden schulischen Qualifikationen (also mit Hauptschul- oder fehlendem Schulabschluss), für Frauen sowie für Ausbildungssuchende mit Migrationshintergrund besonders hoch. Als benachteiligt werden dabei Personen aufgefasst, die im Vergleich zu ihren Altersgenossen bei der Versorgung mit Ausbildungsplätzen im Nachteil sind (BIBB 2010: 251). Das Alter der Ausbildungsplatzsuchenden wurde bisher jedoch selten als eigenständiger Risikofaktor des Übergangs in Ausbildung bedacht und untersucht. Dies erstaunt, denn inzwischen liegen hierzu einige bemerkenswerte empirische Befunde vor.

Das Mindestalter für den Eintritt in die duale Berufsausbildung ist institutionell durch den Beginn der Schulpflicht, die Vollzeitschulpflicht sowie arbeitsgesetzliche Bestimmungen geregelt. Es liegt sowohl in der Schweiz als auch in Deutschland bei ca. 16 Jahren. ${ }^{1}$ Bildungsstatistische Daten belegen jedoch eine zunehmende Verzögerung des Ausbildungsbeginns, der heute in beiden Ländern deutlich über der institutionell vorgegebenen Altersnorm liegt. Das

\footnotetext{
${ }^{1}$ Die Vollzeitschulpflicht, die - je nach Bundesland - bis zum Abschluss des 9. oder 10. Schuljahres dauert, sowie der Beginn der Schulpflicht zwischen dem fünften und siebten Altersjahr regeln in Deutschland und der Schweiz die untere Altersgrenze eines möglichen Eintritts in die duale Ausbildung. Dazu kommen arbeitsgesetzliche Bestimmungen zum Mindestalter beim Abschluss von Ausbildungsverträgen (Deutschland: 15 Jahre; Schweiz: 16 Jahre).
}

Durchschnittsalter aller Ausbildungsanfängerinnen ${ }^{2}$ lag im Jahr 2006 in der Schweiz bei 18 Jahren (Ohne Autor 2008) und in Deutschland bei 19,3 Jahren (Beicht et al. 2007). Das im Durchschnitt höhere Alter der Ausbildungsanfänger in Deutschland dürfte sich durch den im Vergleich zur Schweiz größeren Lehrstellenmangel der vergangenen Dekade und der damit zusammenhängenden Expansion des Übergangssystems erklären (ausführlicher dazu Seibert et al. 2009: 598).

Während sich der Beginn der Ausbildung für Jugendliche zunehmend verzögert, bekunden auch ältere Lehrstellensuchende Probleme bei der Suche nach einem Ausbildungsplatz. Sowohl in Deutschland als auch in der Schweiz sinken nach mehr als zweijähriger Ausbildungsplatzsuche die Chancen auf eine betriebliche Ausbildung markant (Beicht und Eberhard 2009: 89; Hupka-Brunner et al. 2010: 22f.). In der Schweiz betrifft das bereits Jugendliche, die älter als 19 Jahre sind, Für Deutschland ist bekannt, dass Bewerber, die über 20 Jahre alt sind, erheblich schlechtere Chancen haben, einen Ausbildungsplatz zu finden (BIBB 2010: 80).

Somit lassen sich zwei auf den ersten Blick konträre Sachverhalte konstatieren: Während die Ausbildungsbetriebe immer weniger jüngere Auszubildende einstellen, finden etwas ältere Bewerber nur schwer einen Ausbildungsplatz. Die Verzögerung des Eintritts in die duale Ausbildung bei gleichzeitig verminderten Chancen, ab einem gewissen Alter noch einen betrieblichen Ausbildungsplatz zu erhalten, bedeutet für die Ausbildungssuchenden ein limitiertes biographisches Zeitfenster für einen erfolgreichen Eintritt in eine Ausbildung.

Ziel des vorliegenden Artikels ist es, theoretisch sowie empirisch zu klären, ob und wie die bildungsstatistisch dokumentierten unteren und oberen Altersgrenzen für den Übergang in die Ausbildung mit der Ausbildungsplatzvergabe der Betriebe zusammenhängen. Dabei interessiert, aus welchen betrieblichen Beweggründen das Bewerbermerkmal Alter, welches die Selektionsverantwortlichen bereits den Bewerbungsdossiers entnehmen können, bei der Auswahl der Auszubildenden berücksichtigt wird. Der Autor schlägt vor, die betrieblichen Motive mit der französischen Soziologie der Konventionen zu theoretisieren. Altersangaben könnten - so die theoretische Annahme - auf dem Hintergrund selektionsrelevanter Konventionen der Arbeit interpretiert werden, den wesentlichen Beziehungs- und Koordinationsprinzipien in Arbeits- und Ausbildungsorganisationen. Diese Prinzipien gewährleisten die Abstimmung der gegenseitigen Erwartungen und Anforderungen zwischen den betrieblichen Akteuren und den (zukünftigen) Auszubildenden, weshalb sie im Selektionsprozess die Beurteilung der Bewerber anleiten. Als Rechtfertigungsprinzipien

\footnotetext{
${ }^{2}$ Mit männlichen oder weiblichen Gruppenbezeichnungen sind nachfolgend jeweils beide Geschlechtergruppen gemeint, sofern der Kontext nicht auf eine bestimmte Gruppe schließen lässt.
} 
ermöglichen sie zugleich die Begründung und Legitimation der Personalauswahl. Falls sich das Alter aus betrieblicher Sicht als selektionsrelevant erweist, stellt sich somit die Frage, auf welche Konventionen der Arbeit Betriebe konkret Bezug nehmen, um diesem Auswahlkriterium Sinn zuzuweisen und es damit zu rechtfertigen. Die Daten der Schweizer Studie ,Lehrlingsselektion in kleinen und mittleren Betrieben ' (Imdorf 2010b) ermöglichen eine empirische Klärung dieser Frage.

Der Aufsatz ist folgendermaßen gegliedert: Im Anschluss an einen kurzen Überblick über bisherige Erklärungsansätze der Altersdiskriminierung bei der Stellen- und Ausbildungsstellenvergabe und ihrer Begrenzungen (Abschn. 2) wird in die konventionensoziologische Betrachtung der Personalselektion eingeführt und die betriebliche Bedeutung des Auswahlkriteriums Alter darin verortet (Abschn. 3). Nach einer kurzen Darstellung der Stichprobe, der erhobenen Daten sowie der Datenanalyse (Abschn. 4) werden die empirischen Ergebnisse aus der Schweiz zu betrieblichen Präferenzen für Bewerber zu Ungunsten von direkten Schulabgängern sowie zu Ungunsten von als zu alt geltenden Bewerbern präsentiert. Der Aufsatz schließt mit einer Diskussion der zentralen Konventionen der Arbeit, mit denen Betriebe die Durchsetzung von Altersgrenzen bei der Vergabe ihrer Ausbildungsplätze rechtfertigen. Abschließend wird das Potential des konventionentheoretischen Interpretationsrahmens für die Analyse der Ausbildungsplatzvergabe reflektiert (Abschn. 5).

\section{Erklärungsansätze für die Relevanz des Alters bei der (Ausbildungs-) Stellenvergabe}

Die Gründe für die hohe Relevanz des Alters für eine erfolgreiche Ausbildungsplatzsuche sind bisher weitgehend unerforscht. Als mögliche Ursachen für die Zunahme ,älterer Ausbildungsanfänger werden unter Anderem konjunkturelle Gründe diskutiert. Eine relative Angebotsverknappung kann einen Rückstau der Lehrstellenbewerber und eine zunehmende Beanspruchung des Übergangssystems bewirken (Erni und Fleischmann 2006; Ulrich und Eberhard 2008). Eine alternative Erklärung betont, dass gestiegene Arbeitsund Ausbildungsanforderungen es für einen Teil der Betriebe als zweckmäßig erscheinen lässt, in einigen Berufen vermehrt volljährige Ausbildungsplatzbewerber zu berücksichtigen. Das höhere Ausbildungsalter erklärt sich in Deutschland hingegen nur zu einem geringen Teil durch eine betriebliche Präferenz für Jugendliche mit Fachhochschuloder Hochschulreife als Auszubildende (Ulrich und Eberhard 2008: 40). Auch in der Schweiz lässt sich die Verzögerung des Übergangs von der Schule in die Ausbildung nicht mit schulischen Nachteilen der ,jüngeren' Bewerber erklären (Erni und Fleischmann 2006).
Für die Schweiz gibt es vereinzelt Hinweise darauf, dass die Betriebe durch ihre Selektionspraxis aktiv eine untere Altersgrenze durchsetzen, die über den institutionell vorgegebenen Altersnormen liegt. Gemäß dem ,Schweizer Lehrstellenbarometer' haben im Frühjahr 2006 12\% der Ausbildungsbetriebe den Wunsch geäußert, dass Schulabgänger zwischen Schulabschluss und Lehrbeginn ein Zusatzjahr absolvieren, dies insbesondere in den Verkaufs- und Heilberufen (BBT 2006: 42). Auch eine Expertenbefragung unter Westschweizer Betrieben hat ergeben, dass ein Trend besteht, zehn und mehr Schuljahre als Basis für den Eintritt in die berufliche Grundbildung zu fordern bzw. ,ältere' Lehrstellenbewerber zu bevorzugen (Erni und Fleischmann 2006). Insbesondere für Berufe mit Kundenkontakt (z.B. im Einzelhandel) verbinden die Betriebe mit einem höheren Eintrittsalter eine höhere ,Reife' der Auszubildenden, die sich in ausgeprägteren Arbeitstugenden und höheren Sozialkompetenzen äußert (ebd.).

Die vereinzelten betrieblichen Stellungnahmen verlangen nach weiteren empirischen Belegen und einem angemessenen Interpretationsrahmen, der erklärt, warum und wie das Kriterium Alter im betrieblichen Auswahlprozess relevant werden kann. Untersuchungen zur altersspezifischen Personalauswahl beziehen sich in der Regel auf ökonomische Erklärungsmodelle. Betriebliche Einstellungsentscheidungen werden zum einen als eine Profit maximierende Antwort auf unterschiedliches Humankapital-Angebot interpretiert. Gemäß der Humankapitaltheorie (Becker 1993) korreliert das Alter einer Person mit deren Bildungsressourcen und Arbeitserfahrung, was eine Verbindung von (höherem) Alter und (höherer) Produktivität erlaubt (GarnerMoyer 2003: 80; Kitching 2006: 877). Auch wenn die Arbeitgeber in der Regel nicht wissen, wie Produktivität und Alter bei einzelnen Bewerbern konkret zusammenhängen, so erlaubt das Alter dennoch eine Abschätzung der Produktivität auf Basis von Erfahrungswerten (im Sinne der statistischen Diskriminierung vgl. Riach und Rich 2007).

Dass altersspezifische Produktivitätsannahmen auch bei der Selektion von Auszubildenden relevant sein könnten, ist angesichts der vergleichbaren Schulbildungsdauer sowie der fehlenden Arbeitserfahrung von Schulabgängern allerdings fragwürdig. Das Problem ökonomischer Erklärungsansätze besteht zudem darin, dass ihre Prämisse, wonach alle Bewerber mit einer individuellen Produktivität bzw. Kompetenz ausgestattet sind, die komplexen sozialen Bezüge eines Betriebs vernachlässigt, die ebenso zum wirtschaftlichen Erfolg beitragen (Kirschenman und Neckerman 1991: 231). Gemeint ist zum einen das soziale Beziehungsgeflecht innerhalb eines Betriebs, d.h. die horizontalen betrieblichen Sozialbeziehungen innerhalb der Belegschaft (Kitching 2006: 877). Modelle der sozialen SchlieBung (Roscigno et al. 2007) gehen davon aus, dass Diskriminierung bei der Stellenvergabe vorwiegend durch parti- 
kularistische Interessen der betrieblichen Belegschaft verursacht ist. Die Personalverantwortlichen respektieren solche Partikularinteressen, wenn sie nach Personen suchen, die sozial möglichst gut zur Belegschaft ,passen', um Kohäsion und Harmonie der bestehenden Arbeitsgemeinschaft zu sichern und sozialen Spannungen vorzubeugen (Hohn 1988). Kitching (2006) berichtet exemplarisch von einem Personalmanager, der auf die Einstellung älterer Mitarbeiter verzichtete, weil er befürchtete, diese würden nicht in die Kultur des jungen Unternehmens passen. Personalverantwortliche sind darüber hinaus daran interessiert, Personen einzustellen, von denen sie erwarten, dass sie sich der betrieblichen Anleitung und Führung weder entziehen noch widersetzen werden, die sich also möglichst reibungsfrei ,managen " lassen (Kitching 2006: 877). Vor diesem Hintergrund werden ältere Bewerber problematisiert, weil sie eventuell nicht gewillt sein könnten, die Autorität junger bzw. jüngerer Vorgesetzter zu anerkennen.

Neben den horizontalen und vertikalen innerbetrieblichen Sozialbeziehungen gilt es auch die betrieblichen Markt- und Kundenbeziehungen bei der Analyse von Altersdiskriminierung zu berücksichtigen. Gemäß Becker (1971: 75-76) kann die Kundschaft bei der Bewertung eines Produkts oder einer Dienstleistung Merkmale des Verkaufspersonals wie Geschlecht, race oder auch das Alter mitberücksichtigen und auf dieser Grundlage ein Angebot meiden. Solche angenommenen Präferenzen der Kunden können die Personalauswahl entsprechend beeinflussen, wie empirische Studien belegen (Kitching 2006: 877f; Sargeant 2006: 83; Riach und Rich 2007).

Das betriebliche Selektionskalkül ,Produktivität lässt sich unter Berücksichtigung solcher Überlegungen auch als Resultat funktionierender und profitabler Sozial- und Kundenbeziehungen am Arbeitsplatz, und nicht ausschließlich als Funktion individueller Kompetenzen im Sinne der Humankapitaltheorie verstehen (Shih 2002: 102). Das Alter von Bewerbern kann angesichts der sozialen Erfordernisse von Ausbildungsbetrieben bei der Vergabe von Ausbildungsplätzen demnach auch als soziologisch bedeutsam erachtet werden. Die Berücksichtigung betriebsrelevanter Sozialbeziehungen erfordert somit ein soziologisches Modell der (Ausbildungs-) Stellenvergabe.

\section{Das betriebliche Auswahlkriterium Alter aus konventionensoziologischer Perspektive}

\subsection{Die Soziologie der Konventionen}

Die bisherigen Erklärungsansätze - Humankapitaltheorie, soziale Schließung und Kontrolle, Kundenbindungsstrategien - verweisen auf unterschiedliche theoretische und disziplinäre Modelle der Personalauswahl. Die französische Soziologie der Konventionen erlaubt deren Integration in ein transdisziplinäres Modell der (Ausbildungs-) Stellenvergabe auf Basis einer pragmatischen, situativen Handlungstheorie. Die Theorie der Konventionen verbindet ursprünglich die Analyse ökonomischer Institutionen mit einer pragmatischen Soziologie (Diaz-Bone und Thévenot 2010) mit dem Ziel, Wirtschaftshandeln als soziales Handeln zu analysieren. Indem die Grenzziehungen zwischen den Wirtschaftswissenschaft und der Soziologie überbrückt werden, soll es nach Eymard-Duvernay et al. (2010) möglich werden, drei Fragestellungen, die in den Wirtschaftswissenschaften traditionell getrennt behandelt werden, zusammen zu bringen: die Frage der Rationalität, der Koordination, sowie des Werturteils für das Wirtschaftshandeln. Die menschliche Rationalität wird zuallererst als interpretativ (und nicht primär als kalkulierend) gedacht. Ein Akteur interpretiert mit Hilfe von konventionsbasierten Kategorien die Situation und das Handeln der Anderen, um seine Handlungen zu koordinieren. Die Konventionen, auf die er sich dabei bezieht, orientieren sich zum Teil an kollektiven, auf unterschiedliche Formen des Gemeinwohls ausgerichtete Werte, die nicht auf individuelle Präferenzen reduziert werden können. Das Forschungsinteresse der Économie des conventions ${ }^{3}$ ist auf diese gelingende Koordination wirtschaftlicher Akteure unter Bedingung von Unsicherheit in Bezug auf die Handlungen und Erwartungen anderer Akteure gerichtet.

Zentral ist dabei die Annahme von Konventionen, die als Reaktion auf solche Unsicherheit entstehen und deren Handhabung ermöglichen (Salais und Storper 1992). Einige der Vertreter der Économie des conventions heben mit Bezug auf Lewis (1969) den Charakter von Konventionen als einen Mechanismus der Bewältigung von Handlungsunsicherheit in Koordinationssituationen hervor. Andere wiederum betonen die kognitive Funktion der Konvention für die Evaluation von Situationen (Diaz-Bone 2009a: 238).

In der ersten Leseart erlauben Konventionen, soziales Handeln unter Unsicherheit in voraussagbarer Art und Weite zu organisieren und zu koordinieren. Gemäss der Definition von Lewis (1969: 42) liegt eine Konvention vor, wenn beim Auftreten eines bestimmten Koordinationsproblems jedes Gruppenmitglied (a) einer bestimmten Regularität folgt und (b) von jedem anderen Gruppenmitglied erwartet, dass es das gleiche tut, sowie (c) jedes Gruppenmitglied es vorzieht, der Regularität zu folgen, sofern auch die anderen es tun, weil so eine Lösung des Koordinationsproblems erreicht werden kann. Einmal etabliert ermöglicht eine Konvention nach Lewis ein stabiles, sich selbst perpetuierendes System von Präferenzen, Erwartungen und Hal-

\footnotetext{
${ }^{3}$ Économie des conventions (,Ökonomie der Konventionen') ist die ursprüngliche Bezeichnung des hier präsentierten Ansatzes. Obwohl in einem interdisziplinären Feld entwickelt, hat der Ansatz inzwischen in der (Wirtschafts-)Soziologie einen besonders großen Einfluss, weshalb Diaz-Bone und Thévenot (2010) für die Bezeichnung ,Soziologie der Konventionen" plädieren.
} 
tungen, das in der Lage ist, unbegrenzt lange fortzubestehen (ebd.). Konventionen beinhalten demnach ein geteiltes (stillschweigendes oder bewusstes) Einverständnis darüber, auf welcher normativen Basis kollektives Handeln realisiert werden soll (Biggart und Beamish 2003). ${ }^{4}$ Wenn Interaktionen erfolgreich und immer wieder in vergleichbaren Situationen erfolgen, dann werden die Handlungsmuster zu inkorporierten Routinen (Storper und Salais 1997: 16).

In der zweiten Leseart dienen Konventionen als selbstverständliche Schablonen zur Interpretation von Situationen sowie zur Beurteilung und Planung situationsangemessener Handlungen. Im Sinne soziokultureller Schemata tragen sie nun zur Bildung einer gemeinsamen Perspektive bei, um künftige Friktionen und Konflikte in der gegenseitigen Interaktion zu vermeiden und kollektives Handeln zu koordinieren (Dodier 2010). ${ }^{5}$ In dieses zweite Verständnis von Konventionen lässt sich auch der Beitrag von Luc Boltanski und Laurent Thévenot einordnen, die mit dem Grundlagenwerk ,De la justification“ (deutsche Übersetzung: Boltanski und Thévenot 2007) den transdisziplinären Ansatz der Économie des conventions mitbegründet haben. Ihr Beitrag hebt den Wert von Konventionen als Rechtfertigungsordnungen und -ressourcen für die Schlichtung alltäglicher Konflikte hervor. Weil Konventionen damit auch die Legitimation von Entscheidungen ermöglichen, ist die Rechtfertigungstheorie von Boltanski und Thévenot für das weiter unten skizzierte Modell der Ausbildungsplatzvergabe von besonderer Bedeutung.

Boltanski und Thévenot haben dabei verschiedene Wertigkeitsordnungen und Rechtfertigungsprinzipien des Wirtschaftssystems (Konkurrenz, Effizienz, Tradition, Solidarität u.a.) ausführlich beschrieben. Die Économie des conventions bezieht das Konzept der Konvention darauf und entnimmt ihrer Rechtfertigungstheorie gleichzeitig das pragmatische Modell eines Akteurs, der über die reflexive Kompetenz verfügt, Probleme der Handlungskoordination in Arbeitsorganisationen durch eine Bezugnahme auf Konventionen zu bewältigen (Diaz-Bone 2009a: 236). ${ }^{6}$ Die Be-

\footnotetext{
${ }^{4}$ Konventionen werden dabei nicht als dem Handeln äußerliche Institutionen gedacht. Sie sind in das Handeln vielmehr, eingelagert‘. Sie entstehen als pragmatische Lösungen zunächst situativ und können sich erst von da aus verfestigen (Diaz-Bone 2009b: 39). Die Konventionentheorie wird dennoch als eigenständiger institutionentheoretischer Ansatz betrachtet. Institutionen werden als Bündel von verstetigten Konventionen aufgefasst, welches als pragmatische Lösung ökonomischer (oder anderer) Probleme entstanden ist und seither als normal gilt (Biggart und Beamish 2003: 458).

${ }^{5}$ Der hier vertretene Konventionsbegriff unterscheidet sich von einer einfachen Sitte im Weberschen Sinne oder von einem fait social im Sinne Durkheims (Diaz-Bone und Thévenot 2010). Im Gegensatz dazu sucht das Konzept der Konventionen nach Querverbindungen zwischen universalistischen, kulturellen und pragmatischen sozialwissenschaftlichen Modellen der sozialen Koordination (Dodier 2010).

${ }^{6}$ Damit vertritt die Theorie ein Akteurmodell, das dem hier interessierenden betrieblichen Selektionsgeschehen in besonderem Maße ge-
}

griffe ,Rechtfertigungsordnung', ,Wertigkeitsordnung ' und ,Konvention' repräsentieren dabei gleichermaßen das zentrale Konzept der Économie des conventions, auch wenn sie jeweils verschiedene Aspekte hervorheben (Diaz-Bone 2009b: 39).

\subsection{Ein konventionensoziologisches Modell der Personalselektion}

Die Soziologie der Konventionen bietet einen umfassenden heuristischen Rahmen für eine empirische Untersuchung der Frage, weshalb sich Betriebe bei der Ausbildungsstellenvergabe neben individuellen Leistungsmerkmalen auch für die Altersangaben der Bewerber interessieren. Das hier vertretene Selektionsmodell (vgl. ausführlicher dazu Imdorf 2008: 119ff) nutzt diesen Rahmen, um die Ausbildungsplatzvergabe aus betrieblicher Perspektive umfassend zu verstehen und gleichzeitig die Grenzen ökonomischer Modelle zu überwinden, die die Relevanz betrieblicher Sozialbeziehungen für die Personalauswahl unberücksichtigt lassen. (Ausbildungs-) Betriebe stellen konventionensoziologisch kompromissfähige Einrichtungen dar, welche die multiplen, teilweise konfligierenden Koordinationsprinzipien von Arbeitsorganisationen vermitteln und integrieren (Thévenot 2001: 411). Personalpolitik stellt dabei ein Mittel dar, die Spannungen zwischen den verschiedenen Konventionen im Betrieb zu kompensieren und damit zu handhaben.

Ein Hauptproblem bei der Auswahl von Auszubildenden besteht darin, unter Bedingungen von Zeitknappheit und zum Teil fehlendem Personalfachwissen, also in Situationen von großer Unsicherheit, das betriebliche Ausbildungsstellenprofil auf pragmatische Weise mit meist unbekannten jugendlichen Bewerbern zu matchen. Die hinsichtlich der schulischen, beruflichen, arbeitsförmigen und sozialen Passung multidimensionalen Koordinationsanforderungen eines Ausbildungsplatzes kann kein Bewerber annähernd perfekt erfüllen. Weil ,der perfekte Kandidat' nicht gefunden werden kann brechen Betriebe ihre Suchbemühungen frühzeitig ab, sobald ein Kandidat gefunden ist, der die multiplen Anforderungen einigermaßen befriedigt (im Sinne des satisficing, vgl. Simon 1957: 169). Das heißt es wird zugunsten eines Kandidaten entschieden, von dem erwartet werden kann, dass er im Rahmen des Ausbildungsverhältnisses keine anhaltenden betrieblichen Störungen hervorrufen wird. Das matching von Bewerber und Stelle erfordert darüber hinaus, dass das Resultat der Selektion in den betriebsrelevanten Öffentlichkeiten auf Akzeptanz stößt. Das

recht wird, da es die (betriebs-) politische Urteilsfähigkeit von Personen betont. Als Akteure gelten in den nachfolgenden Analysen die Selektionsverantwortlichen, die mikropolitische Entscheide fällen und rechtfertigen. 
kritikfähige Publikum eines Kleinbetriebs setzt sich einerseits aus der Kundschaft und den Geschäftspartnern zusammen. Gleichzeitig gilt es auch Protest ,innerhalb der eigenen Mauern', also unter den Mitarbeitern zu vermeiden, um deren Kooperation und Arbeitsmotivation mittelfristig zu sichern. Um vor diesen Öffentlichkeiten legitime Personalentscheide zu treffen, bedürfen Betriebe geeigneter Formen der Rechtfertigung.

Boltanski und Thévenot (2007) haben verschiedene Rechtfertigungsordnungen rekonstruiert, die das Wahrnehmen, Urteilen und Handeln situativ mit Sinn ausstatten und es Personalverantwortlichen ermöglichen, ihre Entscheide zu legitimieren. Damit werden Konventionen bei der Personalauswahl nicht nur als entscheidungsleitend aufgefasst; sie ermöglichen gleichzeitig die Rechtfertigung der Auswahl. Eine als fair erachtete (Personal-) Entscheidung wird dabei durch Konventionen ermöglicht, die das Wohl eines Gemeinwesens befördern (Imdorf 2010a: 203). Mit Boltanski und Thévenot (2007: 93ff) lassen sich verschiedene Gemeinwesen unterscheiden, die jeweils auf einer spezifischen Koordinations- und Rechtfertigungspraxis des menschlichen Zusammenlebens basieren, und die sich durch eine je eigene Wertigkeitsordnung auszeichnen (Diaz-Bone 2009a: 239). Sobald ein Gemeinwesen eine eigene Objektivität reklamiert, die es gestattet, die Wertigkeit bzw. ,Qualität' einer Person zu ermitteln, sprechen Boltanski und Thévenot (2007) von einer ,Welt‘. Die Konvention einer solchen Welt dient nun als eine Äquivalenznorm, die es erlaubt, mehrere Personen miteinander zu vergleichen. Personen, die sich im Hinblick auf die Koordinationsanforderungen einer bestimmten Welt in besonderem Masse bewähren, wird, in der Sprache von Boltanski und Thévenot (2007), ,Größe“ im Hinblick auf diese Welt zugesprochen.

Auf Basis der Soziologie der Konventionen, welche die vielfältigen leistungsbezogenen und sozialen Facetten der betrieblichen Personalselektion angemessen zu integrieren vermag, lässt sich die Bedeutung des Alters im Rahmen betrieblicher Matching- und Rechtfertigungsprozesse nun vertieft untersuchen. Das bereits aus den Bewerbungsunterlagen ersichtliche Alter dient den Selektionsverantwortlichen dabei als kostengünstiges Signal für die erforderlichen Qualität(en) der Ausbildungsstellenbewerber im Hinblick auf die spezifischen Koordinationsanforderungen eines betrieblichen Ausbildungsverhältnisses. Als zentral für die Handlungskoordination in Arbeitsorganisationen des Wirtschaftssystems werden in der Forschungsliteratur gegenwärtig vier Konventionen diskutiert: die industrielle Konvention, die Marktkonvention, die häusliche Konvention sowie die Netzwerkkonvention. Aus Sicht des Ausbildungssystems gilt es zusätzlich die von Boltanski und Thévenot (2007: 254ff) beschriebene Konvention der, staatsbürgerlichen Welt ${ }^{\star} \mathrm{zu}$ berücksichtigen, um die Ausbildungsplatzvergabe angemessen zu verstehen. Im Hinblick auf die Auswahl von Auszubildenden lässt sich nun für jede durch diese Konventionen konstituierten ,Welten' nach der Bedeutung des Alters als Selektionskriterium fragen.

\subsection{Konventionen eines betrieblichen}

Ausbildungsverhältnisses und die Relevanz des Alters ${ }^{7}$

Die industrielle Konvention stärkt das Prinzip der Effizienz in der betrieblichen Handlungskoordination. Um Ressourcen möglichst produktiv zu nutzen, orientieren sich die Handlungsabläufe an einer effizienten Arbeitsorganisation. Die Arbeitsbeziehungen sind planbar und die Arbeitsmethoden und Produkte idealerweise standardisiert. In dieser in ökonomischen Theorien wohlbekannten ,industriellen Welt', in der sich die Qualität der Mitarbeiter an deren ,Produktivität ' misst, ist bei der Selektion von Auszubildenden die Erwartung bedeutsam, dass sie die künftigen Produktionsabläufe aufgrund ihrer Fähigkeiten, Fertigkeiten und körperlichen Voraussetzung unterstützen und nicht stören werden. Anderweitige industrielle Koordinationsanforderungen, an denen Ausbildungsplatzanwärter gemessen werden, sind Arbeitstugenden wie Pünktlichkeit, RegelmäBigkeit oder Ordnung. Hinsichtlich der Bedeutung, die dem Alter bei der Ausbildungsplatzvergabe zugestanden wird, stellt sich die Frage, inwiefern dieses für Produktivitätsannahmen in der industriellen Welt als relevant betrachtet wird.

Die Marktkonvention hebt dagegen die Beziehungsprinzipien des Preises und der Konkurrenz hervor. Die Art der Beziehung zwischen den Akteuren ist strategisch und ihre Koordination orientiert sich auf opportunistische Weise an der Marktlage. Eine Mitarbeiterin gilt in dieser Welt als ,groß', wenn sie ein nachgefragtes Gut mit knappem Angebot bereithält. Für die Auswahl von Auszubildenden bedeutet die Marktkonvention, dass ein künftiger Auszubildender dem Betrieb keine unnötigen Kosten verursachen soll. Zum einen existieren hier betriebliche Überlegungen die nahe legen, keine Jugendlichen einzustellen, die die Ausbildung frühzeitig abbrechen könnten, da dies einer betrieblichen Fehlinvestition gleichkäme (etwa bei unzureichenden schulischen Voraussetzungen, die dazu führen, dass die Mindestanforderungen der Berufsschule nicht erfüllt werden können, vgl. Imdorf 2009). Zum anderen können Auszubildende beim Kontakt mit der Kundschaft aufgrund askriptiver Merkmale (im Sinne der Kundendiskriminierung nach Becker 1971) ihren eigentlichen Marktwert erhalten. Im Hinblick auf die Erfordernisse dieser, Welt des Marktes' gilt es zu fragen, ob Ausbildungsbetriebe bei Bewerber eines bestimmten Alters einen Ausbildungsabbruch bereits antizipieren, oder ob das Alter als relevant für die Kundenbindung gesehen wird, weil Kunden Abneigungen gegenüber

\footnotetext{
${ }^{7}$ Die nachfolgende Skizze der Konventionen der Arbeit orientiert sich an Diaz-Bone (2009a: 242f.).
} 
besonders ,jungen“ oder aber ,älteren` Auszubildenden haben könnten.

Basierend auf der häuslichen Konvention erlangt eine Person, Qualität', wenn ihr aufgrund von sozialer Nähe, Bekanntschaft, Verwandtschaft oder persönlicher Beziehungen Vertrauen entgegengebracht wird. Die Beziehungserwartungen orientieren sich an den Koordinationsformen der traditionell strukturierten Familie, etwa an altersspezifischen Autoritäts- und Abhängigkeitsverhältnissen. Die ,Betriebsfamilie' vermittelt die Loyalität der Mitarbeiter gegenüber der Organisation und stabilisiert damit die soziale Basis eines Betriebs (Hohn und Windolf 1988: 187). Diese soziale Kohäsion ist Voraussetzung für eine rationale Konfliktregulierung und damit auch für eine hohe betriebliche Produktivität. Sie kann jedoch durch Altersdifferenzen wie auch durch geschlechtliche oder ethnische Heterogenität empfindlich beeinträchtigt werden (ebd.: 188). Die Selektion von Auszubildenden in dieser ,häuslichen Welt ' ist bestrebt, konfliktfreie horizontale und vertikale Sozialbeziehungen im Betrieb zu garantieren. Es stellt sich deshalb die Frage, inwiefern das Alter von Bewerbern mit antizipierten Störungen solcher innerbetrieblicher Sozialbeziehungen in Verbindung gebracht wird.

Boltanski und Chiapello (2003) konnten zudem eine Netzwerkkonvention in spätmodernen Arbeitsorganisationen rekonstruieren. Diese weist Personen dann, Größe ' zu, wenn sie Netzwerke bilden, über Flexibilität, Mobilität und Risikobereitschaft verfügen und sich als ,projektfähig ' erweisen. Im Unterschied zur familienweltlichen Beziehungsform werden Beziehungen in der netzwerk- bzw. ,projektförmigen Welt ${ }^{*}$ flexibel und befristet gelebt. Dies trifft unter anderem auf Kundenbeziehungen zu, die im Unterschied zu den innerbetrieblichen Sozialbeziehungen nur von mittlerer Dauer sind. Eine gelingende Kundenbindung beruht in diesem Fall nicht auf dem Koordinationsprinzip der marktförmigen Welt, das einer Mitarbeiterin unabhängig ihrer Handlungskompetenz aufgrund eines askriptiven, von der Kundschaft präferierten Merkmals, Größe` zuweist. Insbesondere dann, wenn die Qualität der Kundenbeziehung von adäquaten ,Sozialkompetenzen ' der Mitarbeiterin abhängt, wird ihre ,Qualität' durch die Netzwerkkonvention bestimmt. Das Äquivalenzprinzip für die Beurteilung und den Vergleich von Personen in dieser Welt stellt gemäß Boltanski (2007) unter anderem die Fähigkeit dar, die eigene Isolation zu überwinden und neue Beziehungen mit anderen Akteuren einzugehen. Vom betrieblichen Ausschluss gefährdet sind Personen, ,die sich nicht einzubringen wissen, weil sie (...) nicht kommunizieren können, weil sie verschlossen sind“ (ebd.: 10). Im Hinblick auf das Alter als Auswahlkriterium interessiert hier, ob dieses mit kommunikativen und kontaktfördernden Kompetenzen assoziiert wird, die für das Knüpfen und Erhalten von Kundenbeziehungen als relevant erachtet werden.
Zusätzlich zu den bisher ausgeführten vier Konventionen der Arbeit muss schließlich die für das Ausbildungssystem zentrale staatsbürgerliche Konvention berücksichtigt werden, die auf einem Kollektivinteresse beruht. Diese Konvention reklamiert einen Gesellschaftsvertrag, der das eigensüchtige Handeln der Betriebe durch ein Bekenntnis zur Wahrung des sozialen Friedens einschränkt (Imdorf 2008: 126). Bei der Gestaltung von Beziehungen rückt das Prinzip der Chancengleichheit in den Vordergrund. Da der Staat die betriebliche Lehrlingsauswahl im Unterschied zur Gestaltung des Ausbildungsverhältnisses kaum reguliert, vermag die staatsbürgerliche Konvention die Vergabe von Ausbildungsplätzen in der Regel nur schwach zu strukturieren. Es stellt sich die Frage, ob Betriebe diese Konvention auch freiwillig vertreten und Bewerbern zu einer Ausbildung verhelfen, die aufgrund ihres Alters als benachteiligt eingestuft werden.

Die skizzierten Konventionen der Arbeit und der Ausbildung können als wesentliche Beziehungs- und Koordinationsprinzipien von Ausbildungsorganisationen betrachtet werden. Betriebe lassen sich dabei nach unterschiedlichen Konfigurationen von Konventionen unterscheiden, die für die Koordination ihres sozialen Zusammenhalts von besonderer Bedeutung sind. Diese Konventionen gewährleisten auch die Abstimmung der gegenseitigen Erwartungen zwischen den betrieblichen Akteuren und den (zukünftigen) Auszubildenden. Sie leiten daher die Beurteilung der Bewerber an. Als Rechtfertigungsprinzipien ermöglichen sie zugleich die Begründung und die Legitimation der Personalauswahl.

Im Folgenden soll mit einer qualitativen Studie aus der Schweiz geklärt werden, für welche ,Welten' eines Ausbildungsbetriebs das Alter der Ausbildungsanwärter ein Selektionskriterium darstellt, und welche ausbildungsrelevanten Qualitäten mit den jeweiligen Wertigkeitsordnungen in Verbindung gebracht werden. Ziel der empirischen Analyse ist es, am Auswahlkriterium Alter das Spektrum an selektionsrelevanten Konventionen aufzuzeigen und das vorgeschlagene Modell der Ausbildungsplatzvergabe empirisch abzusichern. Sofern sich eine Relevanz der propagierten Konventionen nachweisen lässt, belegen sie betriebliche Motive, die den Ausschluss von als zu jung oder als zu alt geltenden Auszubildenden zur Folge haben und die eingangs problematisierten Entwicklungen auf dem Ausbildungsstellenmarkt möglicherweise mitbedingen.

\section{Daten und methodisches Vorgehen}

Zur Beantwortung der Forschungsfrage wird auf die Daten des Schweizer Forschungsprojekts ,Lehrlingsselektion in kleinen und mittleren Betrieben` aus dem Jahr 2005 
zurückgegriffen. ${ }^{8}$ Nachfolgend werden Stichprobe, Datensammlung und Auswertungsstrategie, auf denen die in Abschn. 5 präsentierten Resultate beruhen, kurz beschrieben. Ausführliche Angaben zu Merkmalen der Stichprobe sowie zum methodischen Vorgehen finden sich im Methodenbericht der Studie (siehe Imdorf 2010b: Online Ressource 1).

Stichprobe. Es wurde die Ausbildungsplatzvergabe in Deutschschweizer Ausbildungsbetrieben kleiner und mittlerer Größe untersucht, da diese in der Schweiz annähernd 90\% der Ausbildungsplätze stellen. Die Betriebe wurden indirekt über das Nachverfolgen einzelner Bewerbungen von Jugendlichen rekrutiert, die sich ohne Erfolg auf folgende Ausbildungsberufe beworben hatten (in Klammern sind jeweils die Anzahl Betriebe pro Beruf angegeben, die auswertbare Angaben zum Selektionskriterium Alter geliefert haben) ${ }^{9}$ : Dentalassistent/in (12), medizinische/r Praxisassistent/in (10), Automechanikerin oder Automonteur (9), Autolackierer/in (15), sowie kaufmännische Ausbildungen (14). Diese Berufe boten sich einerseits an, weil sie bildungsstatistisch relevante Ausbildungsberufe repräsentieren, die typischerweise in Klein- und Mittelbetrieben angeboten werden. Zum anderen unterscheiden sie sich in ihrem schulischen Anforderungsprofil, in ihrer Geschlechtstypik, sowie in der Zugänglichkeit für Jugendliche mit Migrationshintergrund. ${ }^{10}$ Alle Berufe sind zudem ab dem 16. Altersjahr erlernbar. In der erreichten Betriebsstichprobe sind Kleinbetriebe (mit max. 50 Angestellten) mit annähernd $80 \%$ überproportional vertreten (Kleinbetriebe haben in der Schweiz im Jahr 2005 66\% aller Ausbildungsplätze angeboten). Entsprechend erlaubt die Stichprobe eine Hypothesenbildung für diese Betriebstypen unter Berücksichtigung der gewählten Bereiche (öffentliche und persönliche Dienstleistungen sowie Autoreparatur-Branche).

Datenerhebung. In den rekrutierten Ausbildungsbetrieben führte der Autor mit den Selektionsverantwortlichen halbstrukturierte Experteninterviews (Froschauer und Lueger 2002) durch. Der Interviewleitfaden umfasste, neben einleitenden Informationsfragen zur interviewten Person sowie zum Betrieb, Fragen zur betriebsüblichen Lehrstellenvergabe. In diesem Zusammenhang wurde danach gefragt, welche Voraussetzungen eine erfolgreiche schriftliche Bewerbung erfüllen muss und weshalb diese Voraussetzungen

\footnotetext{
${ }^{8}$ Das im Nationalen Forschungsprogramm ,Integration und Ausschluss' durch den Schweizerischen Nationalfonds geförderte Projekt am Heilpädagogischen Institut der Universität Fribourg untersuchte in der Zeit von 2004-2006 die betrieblichen Logiken bei der Besetzung beruflicher Ausbildungsplätze in Deutschschweizer Betrieben.

${ }^{9}$ Die nachfolgenden Schweizer Berufsbezeichnungen widerspiegeln die gewählten Ausbildungsberufe im Jahr 2004. Inzwischen wurden mehrere dieser Berufe modernisiert und umbenannt.

${ }^{10}$ Anfänglich galt das besondere Interesse der Untersuchung der Frage, inwiefern die Bewerbermerkmale Nationalität, Geschlecht sowie die Schulbiographie bei der betrieblichen Ausbildungsplatzvergabe relevant werden.
}

für den Betrieb von Bedeutung sind. Dabei wurde die Relevanz des Alters als Kriterium der Vorauswahl schriftlicher Bewerbungen explizit erfragt. 60 Betriebe gaben Stellungnahmen zu diesem Thema ab. ${ }^{11}$ In 42 Fällen wurde die untere und in 48 Fällen die obere Altersgrenze thematisiert. Aus der retrospektiven Befragung zu betrieblichen Selektionsprozessen resultieren Rechtfertigungen der Selektionsverantwortlichen. Die organisationstheoretische Prämisse, wonach Organisationen keine Entscheide treffen, die sie später in ihrer Umwelt nicht rechtfertigen können, impliziert dabei eine Abhängigkeit von realen Entscheidungssituationen und nachträglichem Legitimationshandeln (den empirisch greifbaren und analysierbaren Rechtfertigungsreden). Damit lässt sich von Rechtfertigungen teilweise auf vergangene Entscheidungsprozesse schließen. Methodisch kommt es daher nicht darauf an, Rechtfertigungen zu vermeiden, sondern es gilt sie durch das Forschungsdesign und die Befragungsmethoden zu ermöglichen (Radtke 1996: 117). Zum einen setzt bereits die Anwesenheit und Aufmerksamkeit eines ,fremden' Wissenschaftlers die Befragten unter Druck, das eigene Selektionshandeln zu legitimieren. Zum anderen wurde der Rechtfertigungsdruck in der vorliegenden Untersuchung zusätzlich erhöht, indem die Selektionsverantwortlichen nach den Gründen für die Ablehnung von realen, dem Interviewer bekannten Bewerberinnen befragt wurden.

Datenanalyse. Das transkribierte Datenmaterial der Interviews wurde vorerst mittels altersrelevanter Suchbegriffe deduktiv nach Textstellen codiert, die im Hinblick auf die Fragestellungen bedeutsam waren. Die in diesen Textstellen enthaltenen Rechtfertigungsreden wurden dann einer Argumentationsanalyse nach Toulmin (1996) unterzogen, wie sie Radtke (1996) für sozialwissenschaftliche Verwendungszwecke vorgeschlagen hat. Die Argumentationsanalyse ist hier die Methode der Wahl, da Antworten auf die Frage, weshalb das Alter bei der Ausbildungsplatzvergabe als wichtig erachtet wird, in der Regel im Modus von Argumentationen erfolgen, die beim Adressaten die Zustimmung zu einer bestimmten Schlussfolgerung erzeugen sollen (Radtke 1996, 115). In der empirischen Analyse solcher Schlussfolgerungen gilt es, die Prämisse - die sogenannte Schlussregel - zu erschließen, die die Darstellung einer bereits getroffenen Selektion als richtige Entscheidung erlaubt. Mit Bezugnahme auf die Soziologie der Konventionen können die betrieblichen Koordinationsanforderungen als ein geteiltes und plausibles Wissen verstanden werden, auf das sich die Selektionsverantwortlichen im Sinne von Schlussregeln beziehen, wenn sie ihr Entscheidungshandeln nachträglich mit Argumenten untermauern. Das Argumentationsschema von

\footnotetext{
${ }^{11}$ Die Frage nach der Relevanz des Alters wurde erst im Verlauf der Datenerhebung systematisch in den Interviewleitfaden aufgenommen, nachdem sich dessen Bedeutung für die Ausbildungsstellenvergabe herauskristallisiert hatte.
} 
Tab. 1 Positive Beurteilungen eines verzögerten Ausbildungsbeginns und Pro-Argumente nach Branche

\begin{tabular}{|c|c|c|}
\hline Branche & $\begin{array}{l}\text { Positive Beurteilung der } \\
\text { Verzögerung }\end{array}$ & $\begin{array}{l}\text { Pro-Argumente } \\
\text { (Mehrfachnennungen möglich) }\end{array}$ \\
\hline Arzt- und Zahnarztpraxen & in 16 von 17 Fällen & $\begin{array}{l}\text { Persönliche ,Reife` (9) } \\
\text { Förderlich für Patientenbeziehung (6) } \\
\text { Qualität in der ,industriellen Welt` (3) } \\
\text { Schicksale ertragen (2) } \\
\text { Betriebliche Sozialintegration (1) }\end{array}$ \\
\hline Verwaltungsabteilungen von KMU & in 10 von 13 Fällen & $\begin{array}{l}\text { Persönliche ,Reife‘ (9) } \\
\text { Betriebliche Sozialintegration (5) }\end{array}$ \\
\hline Autogaragen und Lackierereien & in 8 von 12 Fällen & $\begin{array}{l}\text { Persönliche ,Reife‘ (6) } \\
\text { Soziales Engagement des Betriebs (1) }\end{array}$ \\
\hline
\end{tabular}

Toulmin (1996) bietet sich dabei als Werkzeug an, um die Argumentations- und Begründungsmuster in Interviewtexten methodengeleitet offen zu legen. ${ }^{12}$ Es erlaubt eine Untersuchung, ob und wie von wahrgenommenen Alterskategorien unter Bezugnahme auf bestimmte Konventionen der Arbeit (im Sinne von Schlussregeln) ${ }^{13}$ auf die Konklusion geschlossen wird, dass eine solcherart klassifizierte Person für eine Ausbildung im untersuchten Betrieb (nicht) in Frage kommt (ausführlicher zum argumentationsanalytischen Vorgehen Imdorf 2010b).

\section{Empirische Resultate}

Die Aussagen der Selektionsverantwortlichen zur Relevanz des Alters bei der Lehrstellenvergabe lassen sich danach unterscheiden, ob sie eine untere oder eine obere Altersgrenze der Selektion implizieren. Abschnitt 5.1 analysiert, inwieweit Ausbildungsbetriebe Präferenz für ,ältere“ Bewerber haben. Abschnitt 5.2 untersucht dagegen betriebliche Präferenzen für ,jüngere‘ Ausbildungsplatznachfrager.

\subsection{Betriebliche Präferenzen für ,ältere ‘ Bewerber zu Ungunsten von direkten Schulabgängern}

Tabelle 1 zeigt, wie sich die 42 Stellungnahmen zur unteren Altersgrenze bei der Ausbildungsstellenvergabe auf die

\footnotetext{
${ }^{12}$ Im Sinne bewährter Lösungen und handlungsleitender Theorien des Alltags lassen sich Argumentationsmuster auch den sozialen Deutungsmustern zuordnen. Die hier präferierte Argumentationsanalyse wird einer Deutungsmusteranalyse vorgezogen, weil sie die Strukturen einer Entscheidung angemessener zu operationalisieren und damit das für die Entscheidung notwendige Begründungswissen präziser zu identifizieren vermag.

${ }^{13}$ Nicht alle Begründungen des Selektionskriteriums Alter im analysierten Interviewmaterial ließen sich klar einer Konvention der Arbeit zuordnen, da zum Zeitpunkt der Datenerhebung noch keine konventionensoziologische Analyse vorgesehen und die Befragungsstrategie entsprechend nicht auf eine systematische Aufdeckung von handlungsregulierenden Konventionen ausgerichtet war. Entsprechend handelt sich bei den nachfolgenden Resultaten um Ergebnisse einer Sekundäranalyse.
}

Branchen verteilen und in wie vielen Stellungnahmen ein verzögerter Übergang in die Berufslehre als positiv beurteilt wurde. Die dritte Spalte gibt einen Überblick über die wichtigsten Argumente, mit denen die positive Beurteilung eines verzögerten Ausbildungsbeginns begründet wurde.

Gemäß der tabellarischen Übersicht wurde in einer Mehrheit der betrieblichen Stellungnahmen (in 34 von 42 Fällen) ein verzögerter Lehrbeginn als positiv bewertet. Eine solche Wertung war in den untersuchten Arzt- und Zahnarztpraxen besonders ausgeprägt. Wie die Liste der Pro-Argumente zeigt, wurde über alle Branchen hinweg mit der gesteigerten ,Reife“ der Jugendlichen bei einem verzögerten Lehrbeginn argumentiert. Die Worte des Geschäftsführers eines Karrosseriewerks [53] ${ }^{14}$ summieren die dominante Sichtweise: „Objektiv sage ich, jedes Jahr, welches ein Jüngling später in die Lehre kommt, macht ihn ein Jahr reifer“.

Zum einen wurde auf die Persönlichkeitsentwicklung während der Pubertät hingewiesen. Personen, die etwas älter als sechzehn seien, hätten ,,einen Teil der Pubertät schon hinter sich“, was ,in einer Lehre noch eine riesige Rolle" spiele (Ausbildungsleiter, Autogarage [41]). Ein wesentlicher Reifesprung wurde dabei wiederholt im Anschluss an das fünfzehnte Altersjahr konstatiert, also ausgerechnet dann, wenn sich die Jugendlichen während des neunten Schuljahres auf Lehrstellen zu bewerben beginnen: „Zwischen sechzehn, frisch ab Schule und siebzehn, das ist einfach eine Welt (...) das sind Sprünge, die sie da machen, die Jugendlichen“ (Hausarzt, Arztpraxis [9]). Daher werden Jugendliche bevorzugt, die nach neun Schuljahren ,noch ein Zwischenjahr, oder entweder ein zehntes Schuljahr oder irgendwie einen Fremdsprachenaufenthalt" machen, da sie sich in diesem Alter, ,noch sehr verändern oder entwickeln“

\footnotetext{
${ }^{14}$ Die betrieblichen Stellungnahmen werden jeweils mit einer $[\mathrm{Be}-$ triebsnummer] den untersuchten Ausbildungsbetrieben zugeordnet. Im Anhang sind in Tab. 3 sämtliche bei der Analyse berücksichtigten 60 Ausbildungsbetriebe nach Betriebsnummer aufgelistet. Tabelle 3 gibt zusätzlich Auskunft zu Betriebsart, Betriebsgröße, Ausbildungsberuf sowie zur eingegangenen Anzahl Bewerbungen pro Lehrstelle.
} 
würden (Geschäftsleiter, Medienvertrieb [34]). Der Verwalter einer Autogarage [45] argumentierte ähnlich und fühlte sich in seiner Meinung durch einen Kollegen bestätigt, dem wiederholt auffiel, dass seine Auszubildenden während eines schulischen Zusatzjahres eine außerordentliche Entwicklung durchgemacht haben. Ein Zahnarzt [11] betonte schließlich die Ambiguität der Beurteilung, junger' Schülerinnen, die dazu verleite, dass er mit seinem Selektionsentscheid jeweils zuwarte:

„Wenn die noch ein Jahr jünger sind, ist es viel schwieriger sie zu beurteilen. In diesem Jahr passiert einfach noch sehr viel. Allzu jung ist schwierig, oder, und ich möchte mich dann einfach jeweils noch nicht entscheiden".

Zum anderen wurde ein Zusatzjahr zwischen Schulabgang und Ausbildungsbeginn mit mehr Lebenserfahrung assoziiert. Jugendliche, die „schon ein wenig älter“ seien, würden ,schon eine Erfahrung mit(bringen), die kommen nicht einfach direkt aus der Schule“ (Personalfachfrau, Zahnarztpraxis [17]). Ein Zahnarzt [16] empfand es zudem als ,angenehmer", jemanden mit siebzehn oder achtzehn Jahren einzustellen (,,sie haben mehr Erfahrung, sie haben mehr Lebenserfahrung"). Er akzeptiert daher gerne Bewerberinnen, die ein zehntes Schuljahr absolviert oder gar eine Lehre abgebrochen haben, sofern dies ihre berufliche Orientierung fördert. Unter ,mehr Lebenserfahrung' lassen sich auch die Argumente zweier (Zahn-) Ärzte [9; 21] subsumieren, die es jeweils schätzen, wenn eine Jugendliche bereits einmal „fort von zu Hause“ gewesen war bzw. „schon mal außerhalb der Familie gelebt“" habe. Als „reif“ befand man auf einer Gemeindeverwaltung [27] Jugendliche, die "gefestigter" seien, klarer wüssten, was sie wollten und sich mehr einsetzten. Als ,unreif“ gilt dagegen, wer ,,irgendwie so kein Ziel hat“, bzw. „dieses Hoch und Runter“, bis ein Auszubildender merken würde, „,dass er eigentlich muss, und dass nicht wir müssen, dass $e r$ an die Säcke muss" (Gemeindeschreiber-Stellvertreter). Die Stellungnahmen belegen wiederholt, dass eine so verstandene Reife bei Abgängern des 9. Schuljahrs als noch unzureichend herausgebildet beurteilt wird.

Bisher wurde deutlich, dass Informationen zum Alter und zu beanspruchten Angeboten des Übergangssystems bei der Sortierung von Bewerbungsunterlagen verwertet werden, weil sie von den Selektionsverantwortlichen als Hinweise auf die ,Reife' der Jugendlichen - verstanden als Zielstrebigkeit, Selbstverantwortung und Motivation - gedeutet werden. Solche Aussagen lassen jedoch noch keinen Rückschluss auf selektionsrelevante Konventionen der Arbeit zu. Sobald man aber in Interviews explizit nach der betrieblichen Bedeutsamkeit dieser problematisierten Persönlichkeitsentwicklung fragt, lassen sich die Konventionen rekonstruieren, die es im Sinne von Schlussregeln erlauben, von der Annahme einer , ungenügenden altersspezifischen Reife ‘ auf ein problematisches Ausbildungsverhältnis zu schließen und damit ein ,zu junges ' Alter zu einem betriebsrelevanten Selektionskriterium zu erheben. Hinter dem alltagstheoretischen Reifekonzept verbergen sich dabei unterschiedliche Annahmen darüber, wie der altersspezifische Entwicklungsstand der Jugendlichen für ein gelingendes Ausbildungsverhältnis relevant werden könnte.

In zwei Arztpraxen [2;9] wurde darauf verwiesen, dass der Praxisalltag, der die Jugendlichen früh mit Krankheitsbildern und dem Tod konfrontiere, eine gewisse psychische Stabilität - eine Qualität der häuslichen Welt - erfordere. Einzelne Stellungnahmen von Arztpraxen verweisen zudem auf die Bedeutung von ,Reife' in der industriellen Welt der Betriebe, wo eine effiziente, zuverlässige und verantwortungsvolle Verrichtung beruflicher Tätigkeiten verlangt wird. Hingewiesen wurde etwa auf die erforderliche Selbstsicherheit beim Verabreichen von Spritzen (Med. Praxisassistentin [5]), auf die Verantwortungsübernahme, die ein Beruf verlange, ,wo es um Menschenleben gehen kann“ (Med. Praxisassistentin [1]), oder auf die organisatorischen Fähigkeiten, die die telefonische Triage von Patienten in einer Arztpraxis [3] erfordere, wie der folgende Interviewausschnitt belegt:

Interviewer: Was bedeutet das, dass sie [die Bewerberinnen] reif sind?

Medizinische Praxisassistentin: Ja, ein gewisses Auftreten, nicht allzu unsicher. (...) stimmlich ist auch noch ein Kriterium

Ärztin: Ja, das Telefon ist wichtig, oder

Medizinische Praxisassistentin: Ziemlich, ja. Eine hatte zum Beispiel eine ganz leise Stimme, bei der haben wir gesagt, das können wir uns nicht vorstellen Ärztin: Sie müssen einfach insofern eine gewisse Reife haben, weil sie Anrufe entgegen nehmen müssen. Und dann müssen sie eine Situation einschätzen können, oder. Ist jetzt das jemand, der eben äh, etwas Dringendes hat und zufrieden ist, wenn er einen Termin in fünf Tagen bekommt? Sie müssen auch mit schwierigen Leuten umgehen können. Manchmal geht es nur schon zehn Minuten bis jemand irgendeinen Termin hat, der ihm passt und so. Oder der ist ungehalten. Und das braucht einfach eine gewisse Reife, eine gewisses Selbstvertrauen auch, dass nicht gerade bei der ersten Kritik jemand sofort am Boden zerstört ist, denke ich.

Das Interviewzitat zeigt zudem anschaulich, dass „eine gewisse Reife" auch für die Kommunikation mit (schwierigen) Patienten als zentral erachtet wird, womit die Qualität, sich befristet und selbstsicher auf Beziehungen mit Klienten bzw. 
Kunden einzulassen, in den Vordergrund rückt. Dem pflichtet auch der Verwalter einer Zahnklinik [13] bei: „Also normalerweise finde ich auch, also, wenn sie ganz jung sind, das bringt nichts. Man muss natürlich sehen, man ist dann auch vom ersten Tag dann auch mit Leuten konfrontiert, Telefon, Patienten, und da finde ich, muss man ein gewisses Alter haben“. Wenn ,eine ein Jahr älter sei“, so ein weiterer Zahnarzt [12], sei sie ,auch rein vom Umgang (her) ein wenig erwachsener“, was ,in unserem Umfeld, mit all diesen Leuten (...) nicht negativ“ ist. Ein anderer Zahnarzt [15] verdeutlichte, was dies im Berufsalltag bedeutet:

„Man muss ein wenig offen (sein), man muss den Kontakt haben, man muss vielleicht einmal einen Patienten setzen, oder du musst vielleicht, wenn er dich mal etwas fragt, Auskunft geben, ohne gerade rot zu werden. Also, es braucht einfach ein wenig Reife“.

Die diversen Stellungnahmen belegen anschaulich, dass ,Reife " in den privaten Arzt- und Zahnarztpraxen eine Qualität für die berufliche Beziehungsarbeit mit den Patienten und ihren Angehörigen darstellt, der die Netzwerkkonvention der Beziehungsgestaltung zu Grunde liegt. Ein ,zu junges“ Alter der Kandidatinnen wird von den Selektionsverantwortlichen als Hinweis gedeutet, dass „die jungen Frauen“ noch nicht imstande sein könnten, mit Patienten selbstsicher (,ohne rot zu werden") zu kommunizieren, mit ihnen den Kontakt zu suchen und offen zu sein. Dagegen enthält das analysiert Material keine Hinweise darauf, dass Betriebe das Alter der Auszubildenden im Hinblick auf funktionierende Kundenbeziehungen vor dem Hintergrund der Marktkonvention problematisieren würden. Es wird nicht befürchtet, dass Kunden einen Betrieb meiden könnten, weil sie sich (im Sinne der Beckerschen Kundendiskriminierung) , am jungen Alter' von Auszubildenden stören.

Von Betrieben, die eine unzureichende ,Reife" von als zu jung geltenden Kandidatinnen im Hinblick auf die Beziehungsarbeit mit den Kunden bzw. Patienten problematisierten, lassen sich solche unterscheiden, die ,ältere' Auszubildende präferieren, weil sie eine konstante Begleitung ihrer Auszubildenden durch eine einzige Betreuungsperson - ein Merkmal der traditionellen häuslichen Welt, die in diesen Betrieben geschwächt ist - nicht gewährleisten können. In diesen Ausbildungsorganisationen erweist sich die Netzwerkkonvention auch für funktionierende innerbetriebliche Sozialbeziehungen als bedeutsam. Exemplarisch war in einer Arztpraxis [10] mit vorwiegend Teilzeitarbeitskräften die Rede davon, dass eine Auszubildende ,schon ein wenig Persönlichkeit“ haben müsse, ,nicht sechzehn oder fünfzehn ist“" und „,sich selbst organisieren kann“, da es mit den vielen Teilzeitleuten unmöglich sei, eine qualifizierte Fachkraft bereitzustellen, die ,konstant die ganze Woche bei einem ist, auch nicht im ersten Lehrjahr". Als problematisch erweisen sich hier als ,unreif‘ bzw. als zu jung geltende Jugendliche, weil sie einer engen, auf einer häuslichen Konvention beruhenden Anleitung und Führung bedürfen, die der Ausbildungsbetrieb gerade nicht anbieten kann oder will. Ähnliches gilt auch für einen untersuchten Ausbildungsverbund [30], in welchem sich mehrere Betriebe ein Ausbildungsverhältnis teilen. Der Ausbildungskoordinator suchte „,so ein wenig reifere, interessiertere Leute (...), weil sie jedes Jahr eigentlich den Betrieb wechseln“, womit er auf die Flexibilität von Auszubildenden verweist. In der projektförmigen Welt eines Ausbildungsverbundes absolvieren Auszubildende ihre Ausbildungszeit nicht mehr an einem einzigen Ort, sondern sie rotieren im Jahresrhythmus zwischen verschiedenen Betrieben. Deshalb sind Personen erwünscht, die in der Lage sind, betriebliche Sozialbeziehungen befristet einzugehen, was eher von, älteren ‘ als von ,jüngeren' Jugendlichen erwartet wird (Imdorf und Leemann 2011). Während im Ausbildungsverbund das Erfordernis nach flexiblen horizontalen Sozialbeziehungen ein Bewerbungshindernis für ,zu junge' Jugendliche bedeutet, wurde im ersten Fall deren Passung in die vertikale soziale Beziehungsstruktur eines Betriebs mit vielen Teilzeitstellen in Frage gestellt. Beide Fälle zeigen, dass in der projektförmigen Welt die Qualität der Auszubildenden an einem Mindestalter festgemacht wird.

Auch in den untersuchten Autogaragen und Autolackierereien gab es mehrere Hinweise darauf, dass die Betriebe einen verzögerten Ausbildungsbeginn begrüßen, weil ältere Jugendliche als ,reifer" erachtet werden. Das Interviewmaterial lässt eine Rekonstruktion dominanter Konventionen, die den jeweiligen Reifeargumenten zugrunde liegen, in dieser Branche jedoch nicht zu. Aufschlussreich war eine zum verbreiteten Reifediskurs alternative Argumentation einer Autolackiererei [50], die sich aus sozialem Engagement der Förderung und Ausbildung benachteiligter Jugendlicher verschrieben hat und aus diesem Grund Bewerber für die engere Auswahl berücksichtigte, die bereits ein schulisches Zusatzjahr gemacht oder eine Lehre abgebrochen hatten. Hier bezog sich der interviewte Geschäftsinhaber auf die staatsbürgerliche Konvention, um ein höheres Alter bei Ausbildungsbeginn zu rechtfertigen.

Fasst man die betrieblichen Stellungnahmen zur Frage des verzögerten Eintritts in die Berufslehre im Anschluss an die obligatorische Schulzeit zusammen, so zeigt sich, dass mancher Ausbildungsbetrieb diese Verzögerung als vorteilhaft für das Ausbildungsverhältnis sowie für die eigene Arbeitsorganisation beurteilt. Hinter dem Argument der ,Rei-

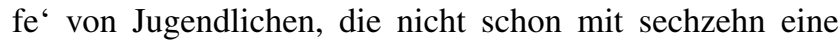
Lehre beginnen, lassen sich dabei in unterschiedlichem $\mathrm{Ma} ß$ die in Abschn. 3.2 ausgeführten Konventionen der Selektion rekonstruieren. Während einige betriebliche Stellungnahmen auf die Bedeutung von ,Reife ' in der industriellen Welt verweisen, sprechen insbesondere die Argumentationen aus den Arzt- und Zahnarztpraxen für die Relevanz von 
Tab. 2 Negative Beurteilungen, älterer' Bewerber und betriebliche Kontra/Pro-Argumente nach Branche

\begin{tabular}{|c|c|c|c|}
\hline Branche & $\begin{array}{l}\text { Negative Beurteilung } \\
\text {,älterer' Bewerber }{ }^{\mathrm{a}}\end{array}$ & $\begin{array}{l}\text { Argumente gegen, ältere“ } \\
\text { Bewerber }\end{array}$ & $\begin{array}{l}\text { Argumente für, ältere } \\
\text { Bewerber }\end{array}$ \\
\hline Arzt- und Zahnarztpraxen & in 10 von 13 Fällen & $\begin{array}{l}\text { Betriebliche Sozialintegration (5) } \\
\text { Risiko Lehrabbruch (3) } \\
\text { Altersnorm (2) }\end{array}$ & $\begin{array}{l}\text { Berufliche Erfahrung (1) } \\
\text { Wille, Motivation (1) }\end{array}$ \\
\hline Verwaltungen von KMU & in 6 von 14 Fällen & $\begin{array}{l}\text { Betriebliche Sozialintegration (3) } \\
\text { Jüngeren eine Chance geben (2) }\end{array}$ & $\begin{array}{l}\text { Soziales Engagement (2) } \\
\text { Sie wissen, was sie wollen (1) }\end{array}$ \\
\hline Autogaragen und Lackierereien & in 6 von 21 Fällen & $\begin{array}{l}\text { Betriebliche Sozialintegration (3) } \\
\text { Risiko Lehrabbruch (2) } \\
\text { Berufl. Sozialisation erschwert (1) }\end{array}$ & $\begin{array}{l}\text { Späte Berufsorientierung (3) } \\
\text { Biographische Erfahrung (3) } \\
\text { Berufliche Erfahrung (2) Kann Auto fahren (2) }\end{array}$ \\
\hline
\end{tabular}

${ }^{a}$ Berücksichtigt wurden Argumentationen, die sich auf Bewerber bis zu einem Alter von 25 Jahren bezogen haben. Die Betriebe haben vereinzelt auch 30-jährige und ältere Bewerber problematisiert, welche die hier interessierende Problemgruppe nicht mehr angemessen repräsentieren

,Reife" für gelingende Kunden- bzw. Patientenbeziehungen in der projektförmigen Welt. Die Netzwerkkonvention dieser Welt strukturiert auch die innerbetrieblichen Sozialbeziehungen von Arbeitsorganisationen, die keine konstante Betreuung eines Auszubildenden anbieten, und in denen das Alter in der Selektion u.a. als Indikator für eine gelingende Sozialintegration und Führbarkeit gewertet wird.

\subsection{Betriebliche Präferenzen zu Ungunsten von als zu alt geltenden Bewerbern}

Wie sich die 48 Stellungnahmen zur Beurteilung von, älteren' Bewerbern auf die Branchen verteilen und wie oft solche Personen in den untersuchten Selektionsprozessen als negativ beurteilt wurden, gibt Tab. 2 wieder. Sie gibt zudem einen Überblick über die wichtigsten Argumente, die gegen sowie für ,ältere' Bewerber bei der Ausbildungsstellenvergabe vorgebracht wurden.

Der Tab. 2 ist zu entnehmen, dass ,ältere ‘ Bewerberinnen in fast der Hälfte der untersuchten Betriebe (in 22 von 48 Betrieben) eingeschränkte Chancen auf einen Ausbildungsplatz haben, insbesondere aber in den untersuchten Arztund Zahnarztpraxen. Das Autogewerbe zeigt sich ihnen gegenüber dagegen offener. Als branchenübergreifendes Argument gegen die Einstellung ,älterer' Bewerber erweist sich die betriebliche Sozialintegration, die bei ihnen in Frage gestellt wird. Befürchtet wird zudem, dass sie ihre Ausbildung abbrechen könnten.

,Ältere' Auszubildende könnten die bewährte formale Hierarchie eines Ausbildungsverhältnisses in Frage stellen, denn in der häuslichen Welt eines Betriebs legitimiert sich die Autorität des Ausbilders qua Alter. So hatte man sich in einer Arztpraxis [1] angesichts der Bewerbung einer über 20-Jährigen, ,die fast gleich alt“ wie die Ausbilderin war, gefragt, ob sich eine solche Person von Letzterer noch ,etwas sagen lässt“. Ein Zahnarzt [19] gab als ,gewisse Reizschwelle“ ein Alter von ,über zwanzig“ bei Lehrbeginn an, da es problematisch werde, ,,sobald die Ausbilderinnen jünger sind" als die Auszubildenden. Der Inhaber einer Autogarage [40] vertrat die Meinung, dass ein Auszubildender die Ausbildung mit fünfundzwanzig abgeschlossen (und somit spätestens mit 22 Jahren begonnen) haben sollte. Damit sei die Bedingung am ehesten gegeben, ,dass jemand den Faden zu mir [dem vorgesetzten Automechaniker] findet". In einem Karrosseriewerk [60] wurde schließlich auf dem Hintergrund einer negativen betrieblichen Erfahrung die Frage des Alters mit dem Geschlecht einer ehemaligen Auszubildenden verknüpft. Der Personalchef argumentierte, dass dieses „Mädchen, das wir angestellt hatten als Lehrling Autolackierer“ mit achtzehn Jahren ,sicher schon mehr Lebenserfahrung“ als „ein Sechzehnjähriger“ mitgebracht habe. Nachdem es mit der jungen Frau während der Ausbildung nach wiederkehrenden Problemen zu einem Disput kam (,da sagte sie: ,Entweder der Malerchef oder ich“ “), wurde das Lehrverhältnis aufgelöst. Der Konflikt wurde mit dem Alter der Auszubildenden gedeutet: „Weil sie eben schon achtzehn war, fand sie, dass sie nicht alles machen müsse als Lehrling" [60].

Solche die Unterordnung und Führbarkeit von Auszubildenden betreffende Argumente gegen ältere Bewerber heben funktionierende vertikale Sozialbeziehungen in der häuslichen Welt als Selektionskalkül hervor. Dagegen lassen anderweitige Stellungnahmen auf die betriebliche Antizipation einer erschwerten sozialen Passung, älterer ' Auszubildender in das horizontale Beziehungsgefüge der häuslichen Welt schließen. Eine Zahnärztin [22] befand, dass Personen, die nach der Schule ,zu viele Sprachaufenthalte“ absolviert hätten und zu viel herumgereist seien, ,wahrscheinlich schwieriger zu integrieren" seien und sich schwer ins Team einfügen ließen. Und der Geschäftsführer eines kleinen InternetDienstleisters [25] mit relativ junger Belegschaft (,wir sind ein relativ junges Team, wir sind im Durchschnittsalter etwa fünfundzwanzig“) sprach sich gegen Bewerbungen aus ,,von 
Leuten, die älter sind als wir selbst“" da man nicht wisse, ,ob das funktionieren würde“.

Einzelnen Stellungnahmen ist zu entnehmen, dass Personalverantwortliche eine gelingende betriebliche Sozialintegration im Rahmen eines Ausbildungsverhältnisses in Frage stellen, sobald die Bewerber von den Ausbildungsverantwortlichen als erwachsen wahrgenommen werden. Eine Gemeindeverwaltung [27] stellte auf dem Hintergrund einer negativen Erfahrung mit einer, älteren ' Auszubildenden nur noch Abgänger des neunten oder zehnten Schuljahres ein:

„Wir haben einmal eine Ältere genommen, und das ging dermaßen in die Hose. Das geht nicht, die passt dann einfach auch nicht in dieses Lehrlingswesen hinein. Die ist abgehoben. Eigentlich ist sie Lehrling und trotzdem schon erwachsen, oder ganz erwachsen, wie man das auch immer nennen will. Und davon sind wir abgekommen (...). Sie war dann auch nicht integriert (...) (Gemeindeschreiber-Stellvertreter, kaufmännische Ausbildung).

Auch der Personalleiter einer zweiten Gemeindeverwaltung [28] war es gewohnt, ,junge Lehrlinge auszubilden“, und er konnte sich ein Ausbildungsverhältnis schwer vorstellen, „,wenn es eine erwachsene Person wäre“. Denn das sei „,sofort wieder ein anderes Verhältnis“", das ,wahrscheinlich das Zwischenmenschliche (...) ein wenig etwas ändern“ würde. Der Besitzer einer Autogarage [42] vermutete in diesem Zusammenhang eher Probleme bei Frauen als bei Männern. Während er sich flexibel zeigen würde, ,wenn ein Dreißigjähriger kommt und eine Lehre machen will“, hat er die obere Altersgrenze bei den Frauen bei „neunzehn“ festgesetzt. Auf dem Hintergrund der Überzeugung, dass „Mädchen natürlich einfach reifer (sind) als die Jungen“ - eine erfahrungsbasierte Annahme, die auch in manchen anderen Betrieben geteilt wird - begründete er seine geschlechterspezifischen Altersbegrenzungen mit dem Argument: „So ein achtzehnjähriges Mädchen ist eine Frau. Ein achtzehnjähriger Junge ist noch ein $B u b^{\prime \prime}$. Damit bezieht er sich auf die familienweltliche Konvention eines Ausbildungsbetriebs, wonach Auszubildende als Kinder zu gelten haben. Nur sie lassen es in der häuslichen Welt eines Betriebs zu, durch erwachsene Ausbilder beruflich und betrieblich geformt $\mathrm{zu}$ werden.

Neben der Infragestellung der vertikalen und horizontalen Sozialintegration im Ausbildungsbetrieb wurde von einigen Betrieben auch vermutet, dass bei ,älteren“ Auszubildenden ein erhöhtes Lehrabbruchrisiko vorliegen könnte, womit ihr betrieblicher Gewinn in der Welt des Marktes in Frage steht. ,Ältere' Bewerber werden manchmal mit ehemaligen Lehrabbrechern gleichgesetzt: „Wenn ja eine mit zwanzig so etwas anfängt, hat sie vorher schon etwa eine zwei [Ausbildungen] abgebrochen. Das ist nicht ein gutes Argument, damit wir sie nehmen“" (Zahnarztpraxis [19]).
Der Zahnarzt antizipiert bei einer solchen Person, ,dass sie etwas, wenn etwas nicht gerade läuft, nicht fertig macht". Auch der Geschäftsführer einer Autolackiererei [58] setzte einen ,älteren' Bewerber mit einem Lehrabbrecher gleich, mit einer Person, ,die den Bettel einfach zu schnell hinschmeißt“, die zu schnell aufgibt, und zu wenig „Biss“ habe, um „diese drei Jährchen“ durchzuziehen. Deshalb nehme man ,lieber einen Jüngeren, der das [den Lackiererberuf] gerade von Anfang an lernt. (...) Und ich sage jetzt einmal, der Schnitt ist zwischen siebzehn achtzehn, so beginnen sie bei uns". Der Ausbilder einer Autogarage mit Lackiererei [55] wurde „,nach oben, so ab zweiundzwanzig [Jahren] schon stutzig“". Er befürchtete, dass die Lehre abgebrochen werden könnte, weil ein solcher Bewerber ,noch nicht weiß, was er überhaupt will“". Vergleichbar argumentierte ein Zahnarzt [20], indem er seine Präferenz für eine junge Auszubildende betonte, ,,die eigentlich einen gradlinigen Weg gemacht hat, und bald weiß, was sie will“. Deshalb würden Bewerbungen von 19- bis 20-Jährigen ,und darüber" wieder zurückgeschickt. In einer Arztpraxis [4] wurde eine ,ältere ' Bewerberin vom leitenden Arzt als ,nicht mehr formbar" eingestuft. „Zu viel Frust (...) und zu viel Konfliktstoff" könnten in einem solchen Fall in einem Lehrabbruch resultieren und ein „gutes Kosten-Nutzenverhältnis“ der Ausbildung in Frage stellen.

Vorbehalte in der häuslichen Welt (insbesondere hinsichtlich sozialer Passung und Führbarkeit) sowie antizipierte Ausbildungsabbrüche in der Welt des Marktes motivieren Ausbildungsbetriebe dazu, bei der Ausbildungsplatzvergabe ,jüngere' Bewerber zu bevorzugen. Es gibt aber auch Betriebe, die, ältere ‘ Bewerber bewusst berücksichtigen. In der industriellen Welt wird etwa deren berufliche Erfahrung und Motivation gewürdigt $[17 ; 21 ; 48 ; 50]$, sowie - im Autogewerbe - der Sachverhalt, dass volljährige Auszubildende Auto fahren könnten, wovon die Garagenbetriebe profitieren [38; 44]. Mehrere Betriebe werten zudem eine wohlüberlegte späte Berufsorientierung als positiv $[25 ; 37 ; 48 ; 51]$ und drei Autolackierer [46; 49; 57] äußerten vor dem Hintergrund ihrer eigenen verzögerten Ausbildungsbiographie Verständnis für ,ältere' Bewerber. Zwei Betriebe [36; 49] begründen die Berücksichtigung, älterer' Bewerber schließlich mit dem sozialen Engagement des Betriebs, also unter Bezugnahme auf die staatsbürgerliche Konvention. Das Aufrufen dieser Konvention ermöglicht jedoch keine klare Vorhersage des Selektionsentscheids von Betrieben. So plädierte der Leiter eines Karrosseriewerks [52] unter Berufung auf die gleiche Konvention für die Bevorzugung jüngerer Kandidaten: „Weil die Schulabgänger, (...) die haben die erste Chance, weil derjenige, der eine (Ausbildung) abbricht, der nimmt jemandem die Chance weg, einzusteigen“. Mit der staatsbürgerlichen Konvention wurde zudem in den Verwaltungsabteilungen zweier Produktionsbetrieben [26; 29] gegen ,ältere` Bewerber argumentiert, die sich für 
eine Zweitausbildung beworben hatten. Diese Betriebe wollten lieber jüngeren Jugendlichen eine Chance zur Erstausbildung geben.

\section{Konklusion}

Im vorliegenden Artikel wurde untersucht, ob und weshalb Ausbildungsbetriebe das Alter von Bewerbern um eine Ausbildungsstelle als Selektionskriterium verwenden. Dabei interessierte, auf welche Konventionen der Arbeit - im Sinne zentraler Beziehungs- und Koordinationsprinzipien von Arbeits- und Ausbildungsorganisationen - sich Ausbildungsbetriebe beziehen, um aus Altersangaben in Bewerbungsdossiers auf ausbildungsrelevante Qualitäten von Bewerbern zu schließen und damit das Alter als Selektionskriterium zu rechtfertigen.

Die Rechtfertigungsreden der Selektionsverantwortlichen haben gezeigt, dass die untersuchten Ausbildungsbetriebe des Kleingewerbes mehrheitlich Bewerber befürworten, die nicht direkt von der Schule abgegangen sind, sondern in der Regel ein Jahr im Übergangssystem verbracht haben. Trotz dieser Präferenz für ,ältere` Bewerber hat sich eine beachtliche Minderheit der Betriebe gegen Bewerber ausgesprochen, die ein bestimmtes Alter bereits überschritten haben (im untersuchten Schweizer Kontext handelt es sich oft um Personen, die älter als 19-jährig sind). Dabei zeigen sich auffällige Branchenunterschiede: Für die untersuchten Arzt- und Zahnarztpraxen des persönlichen Dienstleistungsgewerbes konnte eine ausgeprägte Ablehnung von ,zu jungen ' und ,zu alten' Auszubildenden belegt werden. Im Vergleich dazu besetzt das Gewerbe der Autoreparaturbetriebe seine Ausbildungsplätze altersheterogener.

Bei der inhaltlichen Begründung des Alters als Auswahlkriterium von Auszubildenden rechtfertigen die Betriebe die Durchsetzung von unteren und oberen Altersgrenzen mit Bezugnahme auf spezifische Konventionen der Arbeit. Jugendlichen, die nicht schon mit sechzehn, dem gesetzlichen Mindestalter, eine Lehre beginnen, sondern ein bis zwei Jahre später, gelten aus Sicht mancher Betriebe als ,reifer'. Mit ,Reife' assoziieren die Ausbildungsverantwortlichen mit Bezug auf die Netzwerkkonvention Qualitäten, die insbesondere der projektförmigen Welt ihrer Betriebe zugute kommen. ,Reif" sind demnach Jugendliche, die es verstehen, sich flexibel und kontaktfreudig auf Patientenund Kundenbeziehungen einzulassen sowie jene, die unter arbeitsorganisatorischen Bedingungen, die keine konstante Anleitung und Betreuung ermöglichen, genügend Selbstorganisation bei gleichzeitiger ,Führbarkeit" versprechen. Die Bedeutung des Alters für die projektförmige Welt von Ausbildungsbetrieben wurde insbesondere in den untersuchten
Arzt- und Zahnarztpraxen sowie in den kaufmännischen Abteilungen deutlich. Dagegen gab es in den Reparaturbetrieben des Autogewerbes kaum Hinweise auf die Selektionsrelevanz der Netzwerkkonvention im Zusammenhang mit dem Alter.

Die betrieblichen Rechtfertigungen gegen eine Anstellung, älterer' Lehrstellensuchender beziehen sich dagegen auf zwei andere Konventionen der Arbeit. Die untersuchten Betriebe äußerten teilweise Vorbehalte gegenüber ,älteren Auszubildenden, weil diese als schwer integrier- und führbar gelten. Es wird befürchtet, dass ihre Sozialintegration in der häuslichen Welt der Betriebe misslingen könnte (häusliche Konvention). Dies kommt auch in einer anekdotischen Stellungnahme eines deutschen Geschäftsführers bei Erni und Fleischmann (2006) zum Ausdruck, wonach Betriebe an ,älteren' Bewerbern kritisieren würden, dass sich diese nicht mehr ,formen' ließen. ,Ältere' Personen auf Ausbildungsstellensuche stehen zudem im Verdacht, dass sie die Ausbildung nicht durchhalten könnten. Betriebe unterstellen ihnen mit Bezugnahme auf die Marktkonvention ein erhöhtes Risiko des Ausbildungsabbruchs mit betrieblichen Folgekosten. Beide Rechtfertigungsmodi lassen sich in den untersuchten Kleinbetrieben branchenübergreifend rekonstruieren.

Während das Alter von Ausbildungsstellensuchenden vor dem Hintergrund der häuslichen und der marktwirtschaftlichen Konvention sowie der Netzwerkkonvention eine grundlegende Bedeutung bei der Sortierung von Bewerbungsunterlagen erhält, erweisen sich die Auswirkungen der industriellen und der staatsbürgerlichen Konvention als weniger systematisch. Generell fällt die eher moderate Relevanz des Alters in der industriellen Welt von Ausbildungsbetrieben auf. Offenbar spielt das Alter für einen effizienten und planbaren Einsatz der Auszubildenden im betrieblichen Produktionsprozess eine nachrangige Rolle. Leistungswille, Pünktlichkeit und anderweitige für diese Welt charakteristische Arbeitstugenden scheinen weniger als eine Frage des Alters wahrgenommen zu werden. Die Konvention der staatsbürgerlichen Welt schließlich, die eine Vermeidung von Benachteiligung bei der Ausbildungsplatzvergabe impliziert, wird von den Betrieben flexibel gehandhabt, um sowohl den Einals auch den Ausschluss von als zu jung oder als zu alt geltenden Bewerbern zu rechtfertigen. Einigen Betrieben ist es wichtig, dass, jüngeren' Bewerbern keine Ausbildungsstellen streitig gemacht werden; andere wiederum sind bestrebt, ,älteren ' Bewerberinnen eine Chance zu geben.

Die aufgezeigten Befunde beruhen auf Daten zu mehrheitlich kleinen Ausbildungsbetrieben ausgewählter Branchen. Ob die aufgezeigte Relevanz des Alters auch bei der Vergabe von Ausbildungsplätzen in Großbetrieben oder in anderen Branchen zum Tragen kommt, müsste gesondert 
untersucht werden. Insbesondere die Bedeutung des Alters für die Ausbildungsplatzvergabe in der Großindustrie, die für den deutschen Ausbildungsmarkt bedeutsamer ist als für den schweizerischen, kann hier nicht beurteilt werden. Es gibt aber Hinweise, dass neben Ausbildungsverbünden (Imdorf und Leemann 2011) auch Großbetriebe flexible Auszubildende präferieren (Grob 2004), womit sich die Netzwerkkonvention in diesen Betrieben als bedeutsam erweisen könnte.

Abschließend soll das Potential des konventionentheoretischen Interpretationsrahmens für das Verständnis der Ausbildungsstellenvergabe beurteilt werden. Die Soziologie der Konventionen erlaubt es, die Komplexität von Personalauswahlprozessen angemessen zu erfassen. Sie vermag wesentliche betriebliche Motive und Normen der Selektion zu spezifizieren und stellt damit vorerst einen ertragreichen heuristischen Rahmen für die Sortierung und Interpretation des Interviewmaterials zur Verfügung. Durch die Hervorhebung unterschiedlicher betrieblicher Koordinationsprinzipien ermöglicht der Ansatz eine konsequente Theoretisierung der Personalselektion aus Sicht der Betriebe. Konventionen verweisen somit auf unterschiedliche betriebliche Motive hinter der Verwertung von Altersangaben im Selektionsprozess. Somit lassen sich altersspezifische Übergangsprobleme in die Ausbildung konsequent aus der Perspektive der Ausbildungsbetriebe verstehen.

Die heuristische Natur des verwendeten Ansatzes hat aber auch ihre Grenzen. Bei den propagierten multiplen Welten, die Arbeits- und Ausbildungsorganisationen konstituieren, handelt es sich um komplexe soziale Konstrukte. Sie sind Ausdruck diachroner und lokaler Wirklichkeitskonstruktionen betrieblicher Akteure und als solche nicht einfach zu messen. Um von einem heuristischen Modell zu einer Theorie der Personalselektion zu gelangen, sollte das Modell aber auch einer quantitativen Überprüfung standhalten. Erforderlich wären daher angemessene Indikatoren für die verschiedenen ,Welten " und deren Konventionen. Verschiedene Organisationstypen (Klein- und Großbetriebe unterschiedlicher Branchen, Verbundbetriebe, außerbetriebliche Ausbildungsstätten etc.) ließen sich sodann nach den zentralen Konventionen ihrer Koordination klassifizieren. Dies würde die Untersuchung von Konventionen der Ausbildungsplatzvergabe ermöglichen, die in besonderem $\mathrm{Ma} ß$ zu einer Diskriminierung nach Alter und anderen askriptiven Merkmalen führen.

Damit ist eine theoretische Stärke der Soziologie der Konventionen angesprochen: Sie erlaubt es, Organisationen unter Berücksichtigung ihrer sozialen Existenzbedingungen zu konzipieren. Damit ist sie in der Lage, Phänomene, die alltagssprachlich als Diskriminierung bei der Stellenvergabe bezeichnet werden, aus betrieblicher Perspektive angemessen zu verstehen (vgl. ausführlich Imdorf 2010a). Ge- meinhin wird von Diskriminierung bei der Stellenvergabe gesprochen, wenn ein Arbeits- oder Ausbildungsplatz aufgrund askriptiver Merkmale wie Geschlecht, Nationalität oder Alter und nicht aufgrund der Leistungsfähigkeit einer Person vergeben wird. Dabei bleibt meist ungeklärt, was unter Leistungsfähigkeit konkret zu verstehen ist. In der Regel bezieht sich der Leistungsdiskurs aber implizit auf die Leistungsanforderungen der industriellen Welt einer Arbeitsorganisation. Eine auf diese Welt bezogene Leistungsgerechtigkeit ist dann nicht mehr gegeben, sobald sich bei der Stellenvergabe die Konventionen und die darauf gründenden (Verhaltens-) Erwartungen anderweitiger ,Welten' durchsetzen, also die betrieblichen Kalküle von Sozialintegration, Kundenbindung und risikoarmer Ausbildungsinvestition. Die vorliegenden Befunde belegen, dass Ausbildungsbetriebe das Bewerbermerkmal Alter primär als Indikator im Hinblick auf diese drei Kalküle nutzen, und nur nachrangig als Erwartungswert für die ,industrielle“ Leistungsfähigkeit. Altersdiskriminierung ist damit nicht die Folge einer (unzulässigen) askriptiven Leistungszuschreibung in der industriellen Welt. Sie ist vielmehr das Resultat von Zuschreibungsprozessen in der häuslichen, projekt- oder markförmigen Welt von Arbeits- und Ausbildungsorganisationen.

Wie folgenreich die präsentierten betrieblichen Diskriminierungsprozesse im Hinblick auf die Ausbildungschancen von als zu jung oder als zu alt geltenden Ausbildungsstellenbewerbern tatsächlich sind, kann die vorliegende Studie allerdings nicht beantworten. Dazu sind Bewerberexperimente (Pager 2007; Riach und Rich 2007) erforderlich, mit denen Betriebe, die Ausbildungsstellen anbieten, quantitativ auf Altersdiskriminierung getestet werden können.

\section{Kurzfassung}

Zwei konträre Sachverhalte prägen gegenwärtig die Problematisierung des Alters beim Eintritt in die duale Berufsausbildung: Einerseits stellen Ausbildungsbetriebe zunehmend ältere Auszubildende ein, andererseits erhalten zu alte Bewerber Probleme, überhaupt noch einen Ausbildungsplatz zu finden. Die vorliegende Studie fragt nach den betrieblichen Beweggründen, bei der Auswahl von Auszubildenden die untere Altersgrenze nicht zu tief zu halten und gleichzeitig eine obere Altersgrenze durchzusetzen.

Mit Bezugnahme auf die französische Soziologie der Konventionen und einem daran orientierten Modell der Personalselektion wurde untersucht, ob und weshalb Ausbildungsbetriebe das Alter von Bewerbern um eine Ausbildungsstelle als Selektionskriterium verwenden. Die Sozio- 
logie der Konventionen erlaubt es, die Komplexität von Personalauswahlprozessen angemessen zu erfassen. Durch die Hervorhebung unterschiedlicher betrieblicher Koordinationsprinzipien ermöglicht der Ansatz eine konsequente Theoretisierung der Personalselektion aus Sicht der Betriebe. Dabei interessierte, auf welche Konventionen der Arbeit - im Sinne zentraler Beziehungs- und Koordinationsprinzipien von Arbeits- und Ausbildungsorganisationen - sich Ausbildungsbetriebe beziehen, um aus Altersangaben in Bewerbungsdossiers auf ausbildungsrelevante Qualitäten von Bewerbern zu schließen und damit das Alter als Selektionskriterium zu rechtfertigen. Zur Klärung der Forschungsfrage wurden Experteninterviews mit Ausbildungsverantwortlichen aus 60 Deutschschweizer Kleinbetrieben argumentationsanalytisch ausgewertet.

Die analysierten Rechtfertigungsreden der Selektionsverantwortlichen haben gezeigt, dass die untersuchten Ausbildungsbetriebe des Kleingewerbes mehrheitlich Bewerber befürworten, die nicht direkt von der Schule abgegangen sind, sondern in der Regel ein Jahr im Übergangssystem verbracht haben. Trotz dieser Präferenz für ,ältere ' Bewerber hat sich eine beachtliche Minderheit der Betriebe gegen Bewerber ausgesprochen, die ein bestimmtes Alter bereits überschritten haben. Dabei zeigen sich auffällige Branchenunterschiede: Für die untersuchten Arzt- und Zahnarztpraxen des persönlichen Dienstleistungsgewerbes konnte eine ausgeprägte Ablehnung von,zu jungen' und ,zu alten' Auszubildenden belegt werden. Im Vergleich dazu besetzt das Gewerbe der Autoreparaturbetriebe seine Ausbildungsplätze altersheterogener.

Bei der inhaltlichen Begründung des Alters als Auswahlkriterium von Auszubildenden rechtfertigen die Betriebe die Durchsetzung von unteren und oberen Altersgrenzen mit Bezugnahme auf spezifische Konventionen der Arbeit. Jugendlichen, die nicht schon mit sechzehn, dem gesetzlichen Mindestalter, eine Lehre beginnen, sondern ein bis zwei Jahre später, gelten aus Sicht mancher Betriebe als ,reifer'. Mit ,Reife' assoziieren die Ausbildungsverantwortlichen mit Bezug auf die Netzwerkkonvention Qualitäten, die insbesondere der projektförmigen Welt ihrer Betriebe zugute kommen. ,Reif' sind demnach Jugendliche, die es verstehen, sich flexibel und kontaktfreudig auf Patientenund Kundenbeziehungen einzulassen sowie jene, die unter arbeitsorganisatorischen Bedingungen, die keine konstante Anleitung und Betreuung ermöglichen, genügend Selbstorganisation bei gleichzeitiger ,Führbarkeit' versprechen. Die Bedeutung des Alters für die projektförmige Welt von Ausbildungsbetrieben wurde insbesondere in den untersuchten Arzt- und Zahnarztpraxen sowie in den kaufmännischen Abteilungen deutlich. Dagegen gab es in den Reparaturbetrieben des Autogewerbes kaum Hinweise auf die Selektionsrelevanz der Netzwerkkonvention im Zusammenhang mit dem Alter.
Die betrieblichen Rechtfertigungen gegen eine Anstellung ,älterer' Lehrstellensuchender beziehen sich dagegen auf zwei andere Konventionen der Arbeit. Die untersuchten Betriebe äußerten teilweise Vorbehalte gegenüber ,älteren' Auszubildenden, weil diese als schwer integrier- und führbar gelten. Es wird befürchtet, dass ihre Sozialintegration in der häuslichen Welt der Betriebe misslingen könnte (häusliche Konvention). ,Ältere' Personen auf Ausbildungsstellensuche stehen zudem im Verdacht, dass sie die Ausbildung nicht durchhalten könnten. Betriebe unterstellen ihnen mit Bezugnahme auf die Marktkonvention ein erhöhtes Risiko des Ausbildungsabbruchs mit betrieblichen Folgekosten. Beide Rechtfertigungsmodi lassen sich in den untersuchten Kleinbetrieben branchenübergreifend rekonstruieren.

Während das Alter von Ausbildungsstellensuchenden vor dem Hintergrund der häuslichen und der marktwirtschaftlichen Konvention sowie der Netzwerkkonvention eine grundlegende Bedeutung bei der Sortierung von Bewerbungsunterlagen erhält, erweisen sich die Auswirkungen der industriellen und der staatsbürgerlichen Konvention als weniger systematisch. Generell fällt die eher moderate Relevanz des Alters in der industriellen Welt von Ausbildungsbetrieben auf. Offenbar spielt das Alter für einen effizienten und planbaren Einsatz der Auszubildenden im betrieblichen Produktionsprozess eine nachrangige Rolle. Leistungswille, Pünktlichkeit und anderweitige für diese Welt charakteristische Arbeitstugenden scheinen weniger als eine Frage des Alters wahrgenommen zu werden. Die Konvention der staatsbürgerlichen Welt schließlich, die eine Vermeidung von Benachteiligung bei der Ausbildungsplatzvergabe impliziert, wird von den Betrieben flexibel gehandhabt, um sowohl den Ein- als auch den Ausschluss von als zu jung oder als zu alt geltenden Bewerbern zu rechtfertigen. Einigen Betrieben ist es wichtig, dass ,jüngeren“ Bewerbern keine Ausbildungsstellen streitig gemacht werden; andere wiederum sind bestrebt, ,älteren' Bewerberinnen eine Chance zu geben.

Die Soziologie der Konventionen hat es erlaubt, Arbeitsorganisationen unter Berücksichtigung ihrer sozialen Existenzbedingungen zu konzipieren. Damit ist sie in der Lage, Phänomene, die alltagssprachlich als Diskriminierung bei der Stellenvergabe bezeichnet werden, aus betrieblicher Perspektive angemessen zu verstehen. Die rekonstruierte Altersdiskriminierung bei der betrieblichen Ausbildungsstellenvergabe lässt darauf schließen, dass sowohl der verzögerte Eintritt von Schulabgängern in Ausbildung als auch die Probleme älterer Bewerber bei der Lehrstellensuche durch multiple betriebliche Koordinationsanforderungen mitbedingt sind. 


\section{Executive summary}

Two contrary effects determine how age influences access to apprenticeships: on the one hand, training companies increasingly hire older apprentices. On the other hand applicants who are too old, face difficulty in finding any apprenticeship place at all. The paper investigates the selecting companies' motives in setting both the upper and lower age limits for new recruits using a conceptual framework drawn from the French sociology of conventions.

The sociology of conventions allows the analysis to capture the complexity of firms' personnel selection processes. By highlighting different principles of coordination of the firm this approach permits a comprehensive theorizing of recruitment and selection from the perspective of the firm. Of special interest are the conventions of work and training: the network convention, the domestic convention, the market convention, the industrial convention and the civic convention. These conventions are understood as the central principles of social relations and coordination in work and training organisations. Companies refer to them when they infer training relevant qualities of candidates from age information provided in their application documents. To investigate the research question, expert interviews with personnel managers in 60 small businesses from Germanspeaking Switzerland were interpreted using argument analysis.

The justifications provided by the personnel managers show that most of the analysed small and medium sized training companies favour applicants who have not directly left school, but have usually spent one year in some kind of tiding-over arrangement. Despite this preference for 'older' candidates a significant minority of companies turns down applicants who have already reached a certain age. There are striking sectoral differences: the medical and dental private surgeries investigated in the research rejected both 'too young' and 'too old' apprentices. In contrast, the sector of auto repair shops filled their apprenticeship places more heterogeneously with respect to the age of their apprentices.

Companies, which enforce a lower or upper age limit to select apprentices, justify the use of age as a criterion with reference to specific conventions of work. Young people who do not start their apprenticeship at the legal age of sixteen, but one or two years later, are considered more mature (reif) in the eyes of some companies. Referring to the network convention, the training managers associate 'maturity' with networking qualities. Young people are considered mature if they know how to deal with patients and clients in a flexible and sociable way. Young people are furthermore perceived as mature, when they are self-organized enough to work without constant guidance and individual support but are still young enough to be directed. The importance of age based on the network convention of training companies was particularly apparent in the examined medical and dental surgeries, as well as in the commercial departments of training companies. By contrast, there was little evidence for the importance of this convention in the car repair industry in relation to the age of apprentices.

Personnel managers justify not hiring 'older apprentices' in terms of two different conventions of work. First, they considered that 'older students' were both difficult to integrate and to manage in the work place. The training managers fear that their social integration may fail in the domestic sphere of the companies (domestic convention). Second personnel managers suspect ,too-old' people who are looking for an apprenticeship of not being able to keep up with the training. Referring to the market convention some companies ascribe an increased risk of training dropout to 'older youth' with consequential costs to the firm. Both modes of justification can be reconstructed across different industries in the small businesses under investigation.

It is through the network convention, the domestic convention and the market convention that we see the importance of age as selection criterion for apprenticeships. Other conventions of work contribute less to age discrimination in the hiring of apprentices. Especially the industrial and the civic convention prove to be of less systematic significance. The relevance of age is considered to be only moderate in training companies when it comes to their 'industrial mode' of coordination (industrial convention). Apparently age of applicants is not a reliable predictor of efficient and consistent input at work. Candidates' will to work, punctuality and other perceived work virtues seem to be weighed irrespective of age. The civic convention, finally, which implies equal treatment in hiring, is handled flexibly to justify both the inclusion and the exclusion of applicants considered 'too-young' or 'too-old' respectively. Whereas some companies are concerned that 'young applicants' should get a chance to start an apprenticeship, others rather aim to integrate 'older applicants'.

The sociology of conventions allows for theoretically constructing work organizations by taking into account the social conditions of their reproduction. The approach enables us to understand from a company's perspective phenomena that are colloquially referred to as discrimination in job assignment. The paper concludes that both the school leavers' delayed start of training as well as the problems faced by older job applicants to access apprenticeships are in part caused by training companies and their multiple requirements for social coordination.

Danksagung Der Autor bedankt sich herzlich bei Dirk Verdicchio für das hilfreiche inhaltliche und sprachliche Lektorat sowie bei zwei anonymen Gutachtern und der Herausgeberschaft für die äußerst konstruktive Kritik einer früheren Version des vorliegenden Artikels. 
Anhang

Tab. 3 Untersuchte Betriebe nach Art, Anzahl Mitarbeiter, Ausbildungsberuf, und Anzahl Bewerbungen

\begin{tabular}{|c|c|c|c|c|}
\hline $\begin{array}{l}{[\mathrm{Nr} .]} \\
\text { Betrieb }\end{array}$ & Betriebsart & $\begin{array}{l}\text { Anzahl } \\
\text { Mitarbeiter }\end{array}$ & $\begin{array}{l}\text { Ausbildungsberuf } \\
\text { (offene Lehrstelle) }\end{array}$ & $\begin{array}{l}\text { Anzahl } \\
\text { Bewerbungen }^{\mathrm{a}}\end{array}$ \\
\hline 1 & Arztpraxis & $11-20$ & Medizinische/r Praxisassistent/in & $41-60$ \\
\hline 2 & Arztpraxis & $1-10$ & Medizinische/r Praxisassistent/in & - \\
\hline 3 & Arztpraxis & $1-10$ & Medizinische/r Praxisassistent/in & $41-60$ \\
\hline 4 & Arztpraxis & $1-10$ & Medizinische/r Praxisassistent/in & $26-40$ \\
\hline 5 & Arztpraxis & $1-10$ & Medizinische/r Praxisassistent/in & $16-25$ \\
\hline 6 & Klinik & $21-50$ & Medizinische/r Praxisassistent/in & $101-150$ \\
\hline 7 & Arztpraxis & $1-10$ & Medizinische/r Praxisassistent/in & $26-40$ \\
\hline 8 & Kinderarztpraxis & $1-10$ & Medizinische/r Praxisassistent/in & $26-40$ \\
\hline 9 & Arztpraxis & $1-10$ & Medizinische/r Praxisassistent/in & $26-40$ \\
\hline 10 & Arztpraxis & $1-10$ & Medizinische/r Praxisassistent/in & $101-150$ \\
\hline 11 & Zahnarztpraxis & $1-10$ & Dentalassistent/in & - \\
\hline 12 & Zahnarztpraxis & $21-50$ & Dentalassistent/in & - \\
\hline 13 & Zahnklinik & $21-50$ & Dentalassistent/in & - \\
\hline 14 & Zahnarztpraxis & $1-10$ & Dentalassistent/in & $7-15$ \\
\hline 15 & Zahnarztpraxis & $1-10$ & Dentalassistent/in & $11-15$ \\
\hline 16 & Zahnarztpraxis & $11-20$ & Dentalassistent/in & $26-40$ \\
\hline 17 & Zahnarztpraxis & $1-10$ & Dentalassistent/in & $41-60$ \\
\hline 18 & Zahnarztpraxis & $11-20$ & Dentalassistent/in & $41-60$ \\
\hline 19 & Zahnarztpraxis & $11-20$ & Dentalassistent/in & $26-40$ \\
\hline 20 & Zahnarztpraxis & $11-20$ & Dentalassistent/in & $41-60$ \\
\hline 21 & Zahnarztpraxis & $21-50$ & Dentalassistent/in & $16-25$ \\
\hline 22 & Zahnarztpraxis & $1-10$ & Dentalassistent/in & $101-150$ \\
\hline 23 & KMU Landwirtschaft & $101-250$ & Kauffrau/Kaufmann & $61-100$ \\
\hline 24 & KMU Kunststoffindustrie & $51-100$ & Kauffrau/Kaufmann & $61-100$ \\
\hline 25 & KMU Internet-Lösungen & $1-10$ & Kauffrau/Kaufmann & $26-40$ \\
\hline 26 & KMU Schmierstoffhersteller & $101-250$ & Kauffrau/Kaufmann & $16-25$ \\
\hline 27 & Gemeindeverwaltung & $21-50$ & Kauffrau/Kaufmann & $26-40$ \\
\hline 28 & Gemeindeverwaltung & $11-20$ & Kauffrau/Kaufmann & $16-25$ \\
\hline 29 & KMU Verpackungsindustrie & $101-250$ & Kauffrau/Kaufmann & $41-60$ \\
\hline 30 & Ausbildungsverbund & $21-50$ & Kauffrau/Kaufmann & $>150$ \\
\hline 31 & spezielle Ausbildungsfirma & $101-250$ & Büroassistent/in & - \\
\hline 32 & Kranken- u. Pflegheim & $251-500$ & Kauffrau/Kaufmann & $26-40$ \\
\hline 33 & KMU Fensterbau & $21-50$ & Büroassistent/in & $41-60$ \\
\hline 34 & KMU Medienvertrieb & $1-10$ & Kauffrau/Kaufmann & $61-100$ \\
\hline 35 & Pensionskasse & $51-100$ & Kauffrau/Kaufmann & $61-100$ \\
\hline 36 & Kirchenverwaltung & $11-20$ & Kauffrau/Kaufmann & $16-25$ \\
\hline 37 & Autogarage & $21-50$ & Automonteur/-mechaniker/in & $41-60$ \\
\hline 38 & Autogarage & $11-20$ & Automonteur/-mechaniker/in & $16-25$ \\
\hline 39 & Autogarage & $51-100$ & Automechaniker/in & $61-100$ \\
\hline 40 & Autogarage & $1-10$ & Automonteur/in & $0-6$ \\
\hline 41 & Autogarage & $11-20$ & Automechaniker/in & $7-15$ \\
\hline 42 & Autogarage & $1-10$ & Automonteur/in & $41-60$ \\
\hline 43 & Autogarage & $51-100$ & Automonteur/-mechaniker/in & $>150$ \\
\hline 44 & Autogarage & $1-10$ & Automonteur/in & $101-150$ \\
\hline
\end{tabular}


Tab. 3 (Fortsetzung)

\begin{tabular}{|c|c|c|c|c|}
\hline $\begin{array}{l}{[\mathrm{Nr} .]} \\
\text { Betrieb }\end{array}$ & Betriebsart & $\begin{array}{l}\text { Anzahl } \\
\text { Mitarbeiter }\end{array}$ & $\begin{array}{l}\text { Ausbildungsberuf } \\
\text { (offene Lehrstelle) }\end{array}$ & $\begin{array}{l}\text { Anzahl } \\
\text { Bewerbungen }^{\mathrm{a}}\end{array}$ \\
\hline 45 & Autogarage & $11-20$ & Automonteur/-mechaniker/in & - \\
\hline 46 & Karrosseriewerk & $1-10$ & Autolackierer/in & - \\
\hline 47 & Karrosseriewerk & $11-20$ & Autolackierer/in & - \\
\hline 48 & Autogarage & $21-50$ & Autolackierer/in & $16-25$ \\
\hline 49 & Lackiererei & $1-10$ & Autolackierer/in & $7-15$ \\
\hline 50 & Lackiererei & $11-20$ & Autolackierer/in & $41-60$ \\
\hline 51 & Autogarage & $51-100$ & Autolackierer/in & $41-60$ \\
\hline 52 & Karrosseriewerk & $11-20$ & Autolackierer/in & $101-150$ \\
\hline 53 & Karrosseriewerk & $11-20$ & Autolackierer/in & $61-100$ \\
\hline 54 & Autogarage & $51-100$ & Autolackierer/in & $61-100$ \\
\hline 55 & Autogarage & $101-250$ & Autolackierer/in & $26-40$ \\
\hline 56 & Karrosseriewerk & $21-50$ & Autolackierer/in & $41-60$ \\
\hline 57 & Lackiererei & $1-10$ & Autolackierer/in & $0-6$ \\
\hline 58 & Lackiererei & $1-10$ & Autolackierer/in & $26-40$ \\
\hline 59 & Karrosseriewerk & $11-20$ & Autolackierer/in & $7-15$ \\
\hline 60 & Karrosseriewerk & $11-20$ & Autolackierer/in & $7-15$ \\
\hline
\end{tabular}

${ }^{a}$ Anzahl Bewerbungen für die im Interview diskutierte Lehrstelle (vgl. Spalte Ausbildungsberuf)

\section{Literatur}

BBT - Bundesamt für Berufsbildung und Technologie: Lehrstellenbarometer April 2006. Detaillierter Ergebnisbericht zur Umfrage bei Jugendlichen und Unternehmen. Bundesamt für Berufsbildung und Technologie, Bern (2006)

Becker, G.S.: The Economics of Discrimination, 2. Aufl. University of Chicago Press, Chicago (1971)

Becker, G.S.: Human Capital: A Theoretical and Empirical Analysis with Special Reference to Education, 3. Aufl. Columbia University Press, New York (1993)

Beicht, U., Eberhard, V.: Chancen von Altbewerbern und Altbewerberinnen - Ergebnisse der BA/BIBB-Bewerberbefragung 2008. In: Bundesamt für Berufsbildung (Hrsg.) Datenreport zum Berufsbildungsbericht 2009: Informationen und Analysen zur Entwicklung der beruflichen Bildung, S. 87-92. BIBB, Bonn (2009)

Beicht, U., Friedrich, M., Ulrich, J.G.: Deutlich längere Dauer bis zum Ausbildungseinstieg: Schulabsolventen auf Lehrstellensuche. BIBB Report. Forschungs- und Arbeitsergebnisse aus dem Bundesinstitut für Berufsbildung. H. 2, Sept. 2007 (2007)

BIBB: Bundesinstitut für Berufsbildung: Datenreport zum Berufsbildungsbericht 2010. BIBB, Bonn (2010)

Biggart, N.W., Beamish, Th.D.: The economic sociology of conventions: habit, custom, practice, and routine in market order. Annu. Rev. Sociol. 29, 443-464 (2003)

Boltanski, L.: Leben als Projekt. Prekarität in der schönen neuen Netzwerkwelt. Polar 2, 7-13 (2007)

Boltanski, L., Chiapello, E.: Der neue Geist des Kapitalismus. UVK, Konstanz (2003)

Boltanski, L., Thévenot, L.: Über die Rechtfertigung. Eine Soziologie der kritischen Urteilskraft. Hamburger Edition, Hamburg (2007)

Diaz-Bone, R.: Konvention, Organisation und Institution. Der institutionentheoretische Beitrag der ,Économie des conventions'. Hist. Soc. Res. 34(2), 235-264 (2009a)
Diaz-Bone, R.: Konventionen und Arbeit. Beiträge der ,Économie des conventions ' zur Theorie der Arbeitsorganisation und des Arbeitsmarktes. In: Nissen, S., Vobruba, G. (Hrsg.) Die Ökonomie der Gesellschaft. Festschrift für Heiner Ganßmann, S. 35-56. VSVerlag für Sozialwissenschaften, Wiesbaden (2009b)

Diaz-Bone, R., Thévenot, L.: Die Soziologie der Konventionen. Die Theorie der Konventionen als ein zentraler Bestandteil der neuen französischen Sozialwissenschaften. Trivium 3(5-2010) (Online). http://trivium.revues.org/index3557.html (2010). Eingesehen am 20. Dezember 2010

Dodier, N.: Konventionen als Stützen der Handlung. Elemente der soziologischen Pragmatik. Trivium 3(5-2010) (Online). http://trivium.revues.org/index3565.html (2010). Eingesehen am 24. Juni 2010

Eymard-Duvernay, F., Favereau, O., Orléan, A., Salais, R., Thévenot, L.: Werte, Koordination und Rationalität: Die Verbindung dreier Themen durch die ,Économie des conventions'. Trivium 3(52010) (Online). http://trivium.revues.org/3545 (2010). Eingesehen am 30. Juni 2011

Erni, D., Fleischmann, D.: Genügen neun Jahre nicht mehr? Projekt Nahtstelle obligatorische Schule - Sekundarstufe II. Handlungsbedarf A5 (online). http://www.nahtstelle-transition.ch/files/ nst7122c.pdf (2006). Eingesehen am 10. Oktober 2009

Froschauer, U., Lueger, M.: ExpertInnengespräche in der interpretativen Organisationsforschung. In: Bogner, A., Littig, B., Menz, W. (Hrsg.) Das Experteninterview: Theorie, Methode, Anwendung, S. 223-240. Leske + Budrich, Opladen (2002)

Garner-Moyer, H.: Discrimination et emploi: revue de la littérature. Document d'étude. Etude réalisée pour le compte de la DARES. DARES, Paris (2003)

Grob, M.: Qualifikationsanforderungen an Lehrlinge. Eine quantitative Analyse von Stelleninseraten mit ergänzenden qualitativen Betriebsbefragungen. Lizentiatsarbeit. Soziologisches Institut der Universität Zürich, Zürich (2004)

Hohn, H.-W.: What is qualification? Lifestyle and personality as selection criteria. In: Windolf, P., Wood, S. (Hrsg.) Recruitment and 
Selection in the Labour Market, S. 83-112. Avebury, Aldershot (1988)

Hohn, H.-W., Windolf, P.: Lebensstile als Selektionskriterien - Zur Funktion ,biographischer Signale" in der Rekrutierungspolitik von Arbeitsorganisationen. In: Brose, H.-G., Hildenbrand, B. (Hrsg.) Vom Ende des Individuums zur Individualität ohne Ende, S. 179-207. Leske + Budrich, Opladen (1988)

Hupka-Brunner, S., Sacchi, S., Stalder, B.E.: Social origin and access to upper secondary education in Switzerland: a comparison of company-based apprenticeship and exclusively school-based programmes. Swiss J. Sociol. 36(1), 11-31 (2010)

Hupka-Brunner, S., Gaupp, N., Geier, B., Lex, T., Stalder, B.E.: Chancen bildungsbenachteiligter Jugendlicher: Bildungsverläufe in der Schweiz und in Deutschland. Z. Soziol. Erzieh. Sozial. 31(1), 6278 (2011)

Imdorf, C.: Migrantenjugendliche in der betrieblichen Ausbildungsplatzvergabe - auch ein Problem für Kommunen. In: Bommes, M., Krüger-Potratz, M. (Hrsg.) Migrationsreport 2008. Fakten - Analysen - Perspektiven, S. 113-158. Campus, Frankfurt a.M. (2008)

Imdorf, C.: Die betriebliche Verwertung von Schulzeugnissen bei der Ausbildungsstellenvergabe. Empir. Pädagog. 23(4), 392-409 (2009)

Imdorf, C.: Die Diskriminierung , ausländischer' Jugendlicher bei der Lehrlingsauswahl. In: Hormel, U., Scherr, A. (Hrsg.) Diskriminierung. Grundlagen und Forschungsergebnisse, S. 197-219. VS-Verlag für Sozialwissenschaften, Wiesbaden (2010a)

Imdorf, C.: Forschungsprojekt ,Lehrlingsselektion in KMU`. Methodenbericht. Institut für Soziologie der Universität Basel (Online). http://www.lehrlingsselektion.de/documents/methodenbericht.pdf (2010b). Eingesehen am 10. Januar 2011

Imdorf, C., Leemann, R.J.: Ermöglicht die Flexibilisierung in der Berufsausbildung mehr Chancengerechtigkeit bei der Ausbildungsplatzvergabe? Fallstudie eines Schweizer Ausbildungsverbundes. In: Voss-Dahm, D., Mühge, G., Schmierl, K., Struck, O. (Hrsg.) Qualifizierte Facharbeit im Spannungsfeld von Flexibilität und Stabilität, S. 49-74. VS-Verlag für Sozialwissenschaften, Wiesbaden (2011)

Kirschenman, J., Neckerman, K.M.: We'd love to hire them, but...': the meaning of race for employers. In: Jencks, C., Peterson, P.E. (Hrsg.) The Urban Underclass, S. 203-232. The Brookings Institution, Washington (1991)

Kitching, J.: Can small businesses help reduce employment exclusion? Environ. Plann, C Gov. Policy 24, 869-884 (2006)

Lewis, D.: Convention. Blackwell, Oxford (1969)

Ohne Autor: Mehr als 9 Schuljahre. Zusammenfassender Bericht zum Teilprojekt ,Nahtstelle obligatorische Schule - Sekundarstufe II ${ }^{*}$ (Online). http://www.nahtstelle-transition.ch/files/nst7122e.pdf (2008). Eingesehen am 16. Oktober 2009

Pager, D.: The use of field experiments for studies of employment discrimination: contributions, critiques, and directions for the future. Ann. Am. Acad. Polit. Soc. Sci. 609(January), 104-133 (2007)
Radtke, F.-O.: Wissen und Können - Grundlagen der wissenschaftlichen Lehrerbildung. Leske+Budrich, Opladen (1996)

Riach, P.A., Rich, J.: An experimental investigation of age discrimination in the English labor market. IZA-discussion paper No. 3029, September 2007 (2007)

Roscigno, V.J., Garcia, L.M., Bobbitt-Zeher, D.: Social closure and processes of race/sex employment discrimination. Ann. Am. Acad. Polit. Soc. Sci. 609(January), 16-48 (2007)

Salais, R., Storper, M.: The four ,worlds' of contemporary industry. Camb. J. Econ. 16(2), 169-194 (1992)

Sargeant, M.: Age Discrimination in Employment. Gower, Aldershot (2006)

Seibert, H., Hupka, S., Imdorf, C.: Wie Ausbildungssysteme Chancen verteilen - Berufsbildungschancen und ethnische Herkunft in Deutschland und der Schweiz unter Berücksichtigung des regionalen Verhältnisses von betrieblichen und schulischen Ausbildungen. Köln. Z. Soziol. Soz.psychol. 61(4), 595-620 (2009)

Shih, J.: ,... Yeah, I could hire this one, but I know it's gonna be a problem': how race, nativity and gender affect employers' perceptions of the manageability of job seekers. Ethn. Racial Stud. 25(1), 99-119 (2002)

Simon, H.A.: Models of Man. Mathematical Essays on Rational Human Behavior in Social Setting. Wiley, New York (1957)

Storper, M., Salais, R.: Worlds of Production. The Action Framework of the Economy. Harvard University Press, Cambridge (1997)

Thévenot, L.: Organized complexity: conventions of coordination and the composition of economic arrangements. Eur. J. Soc. Theory 4(4), 405-425 (2001)

Toulmin, S.: Der Gebrauch von Argumenten, 2. Aufl. Beltz Athenäum Verlag, Weinheim (1996)

Ulrich, J.G., Eberhard, V.: Die Entwicklung des Ausbildungsmarktes in Deutschland seit der Wiedervereinigung. In: Beicht, U., Friedrich, M., Ulrich, J.G. (Hrsg.) Ausbildungschancen und Verbleib von Schulabsolventen, S. 13-57. Bertelsmann, Bielefeld (2008)

Christian Imdorf graduated from the University of Fribourg with a social work major and a minor in special education in the year 2000. He received his Ph.D. in educational sciences as a member of the doctorate program 'Shifting Gender Culture' at the Universities of Bern and Fribourg. His dissertation work focused on school qualifications and vocational integration in Switzerland. From 2006 to 2009 he conducted postdoctoral studies at research facilities in Frankfurt, Aix-enProvence and Glasgow. He joined the Institute of Sociology at the University of Basel in 2010 where he holds a SNSF Professorships of the Swiss National Science Foundation (SNSF) since June 2011. His current research interests lie in the selection mechanisms of the education system in general and of the vocational training system in particular. 